\title{
[3+2] versus [4+2] Cycloadditions of Quinone Monoimide with Azadienes: a Lewis Acid-Free Access to 5-Amino-2,3- Dihydrobenzofuranes
}

\author{
Thierry Lomberget, ${ }^{a}$ Fabien Baragona, ${ }^{a}$ Bernard Fenet ${ }^{b}$ \\ and Roland Barret ${ }^{* a}$ \\ ${ }^{a}$ Laboratoire de Chimie Thérapeutique, EA 3741, Ecosystèmes et Molécules \\ Bioactives, Faculté de Pharmacie, Université Claude Bernard Lyon 1, \\ 8 Avenue Rockefeller, 69373 Lyon Cedex 08, France \\ ${ }^{b}$ Centre Commun de RMN, UMR 5012 CNRS, Université Claude Bernard Lyon 1, \\ CPE, 43 Bd du 11 Novembre 1918, 69622 Villeurbanne Cedex, France
}

\section{SUPPORTING INFORMATION}

General. Melting points were measured on a Barnstead Electrothermal 9200 melting point apparatus and are uncorrected. Infrared spectra (IR) were recorded on a Perkin-Elmer FT-IR SPECTRUM ONE spectrometer. Proton nuclear magnetic resonance $\left({ }^{1} \mathrm{H}\right.$ NMR) spectra were recorded on Bruker DRX 300 and DRX 500 Fourier transform spectrometers, using an internal deuterium lock, operating at 300 $\mathrm{MHz}$ and $500 \mathrm{MHz}$ respectively. Chemical shifts are reported in parts per million (ppm) relative to internal standard (tetramethylsilane, $\delta=0.00 ; \mathrm{CDCl}_{3}, \delta=7.26$; acetone- $\mathrm{d}_{6}, \delta=2.05$ ). ${ }^{1}$ Data are presented as follows: chemical shift $(\delta, \mathrm{ppm})$, multiplicity $(\mathrm{s}=$ singlet, $\mathrm{d}=$ doublet, $\mathrm{t}=$ triplet, $\mathrm{q}=$ quadruplet, $\mathrm{m}=$ multiplet, $\mathrm{br}=$ broad), coupling constant (reported in $\mathrm{Hz}$ ), integration, assignment. Carbon magnetic resonance $\left({ }^{13} \mathrm{C}\right.$ NMR) spectra were recorded on Bruker DRX 300 and DRX 500 Fourier transform spectrometers, using an internal deuterium lock, operating at $75 \mathrm{MHz}$ and $125 \mathrm{MHz}$ respectively. The following internal references were used: $\mathrm{CDCl}_{3}(\delta=77.16)$ or acetone- $\mathrm{d}_{6}(\delta=29.84)$. Carbon multiplicities were determined by DEPT or 2D HSQC experiments. Electron-Spray lowresolution mass spectra were recorded on a Thermo ALCQ Advantage. High-resolution mass spectra were recorded on a Thermoquest Finnigan MAT 95 XL spectrometer. Product purification by flash column chromatography was performed using Merck Kieselgel $60 \AA$ (40-63 $\mu \mathrm{m})$. Analytical thin layer chromatography (TLC) was carried using Merck commercial aluminium sheets coated $(0.2 \mathrm{~mm}$ layer thickness) with Kieselgel 60 F254, with visualization by ultraviolet and anisaldehyde stain solution. Absolute ethanol and chloroform (HPLC grade) were used as received without purification.

Quinone monoimide 1a was prepared according to Adams' procedure. $^{2}$

Azadienes 2 were prepared according to literature references. ${ }^{3}$

\footnotetext{
${ }^{1}$ Gottlieb, H. E.; Kotlyar, V.; Nudelman, A. J. Org. Chem. 1997, 62, 7512.

${ }^{2}$ Adams, R.; Looker, J. H. J. Am. Chem. Soc. 1951, 73, 1145.

3 (a) Azadienes 2a,b and 2d-f: Waldner, A. Helv. Chim. Acta 1988, 71, 486. (b) Azadiene 2c: Chaker, L.; Pautet, F.; Fillion, H. Heterocycles 1995, 41, 1169.
} 
Experimental procedure for the equimolar reaction between quinone monoimide 1a and azadiene $2 \mathrm{a}\left(\mathbf{R}^{1}=\mathrm{Me}\right.$ and $\left.\mathbf{R}^{2}=\mathrm{H}\right)$ : preparation of tetracyclic compound 5 and 2,3dihydrobenzofurane $4 a$.

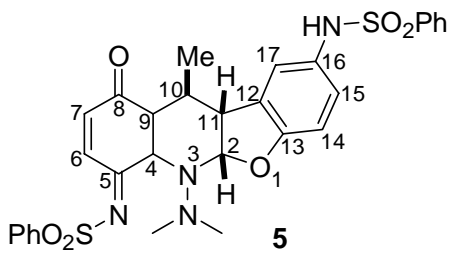

To a solution of quinone monoimide 1a $(495 \mathrm{mg}, 2.0 \mathrm{mmol})$ in absolute ethanol $(190 \mathrm{~mL})$ at $0^{\circ} \mathrm{C}$ was added in one portion a solution of azadiene $2 \mathbf{a}(224 \mathrm{mg}, 2.0 \mathrm{mmol})$ in absolute ethanol $(10 \mathrm{~mL})$. After stirring of the resulting mixture during one hour at $0^{\circ} \mathrm{C}$, the volatiles were removed under high vacuum at $23^{\circ} \mathrm{C}$ (water bath). The pale brown/yellow solid residue was then completely dissolved in ethyl acetate $(\mathrm{ca} 40 \mathrm{~mL})$; addition of petroleum ether $(400 \mathrm{~mL})$ in one portion allowed compound 5 to slowly precipitate (within one hour) as a pale brown powder. The precipitate was collected by filtration on a $5 \mu \mathrm{m}$ Whatmann nylon membrane and washed with a minimum amount of ethyl acetate to afford 5 as a pale yellow finely divided powder $(294 \mathrm{mg}, 24 \%) . \mathrm{Mp} 165-166^{\circ} \mathrm{C}$ (decomposed). ${ }^{1} \mathrm{H}$ NMR $\left(500 \mathrm{MHz}\right.$, acetone- $\left.\mathrm{d}_{6}\right): 1.24\left(\mathrm{~d}, J=7.2,3 \mathrm{H}, \mathrm{CH}_{3}\right), 1.40-1.47(\mathrm{~m}, 1 \mathrm{H}, \mathrm{H1O}), 2.32$ (br s, $6 \mathrm{H}$, $\left.\mathrm{N} M e_{2}\right), 2.86(\mathrm{dd}, J=11.4-5.2,1 \mathrm{H}, H 11), 3.08$ (t, $\left.J=2.2,1 \mathrm{H}, H 9\right), 4.26(\mathrm{t}, J=2.2,1 \mathrm{H}, H 4), 5.67$ (d, $J$ $=5.2,1 \mathrm{H}, H 2), 6.52(\mathrm{~d}, J=10.4,1 \mathrm{H}, H 7), 6.62(\mathrm{~d}, J=8.5,1 \mathrm{H}, H 14), 6.92(\mathrm{dd}, J=8.5-2.2,1 \mathrm{H}, H 15)$, $7.05(\mathrm{~d}, J=2.2,1 \mathrm{H}, H 17), 7.50\left(\mathrm{t}, J=7.5,2 \mathrm{H}, \mathrm{SO}_{2} P h\right), 7.59\left(\mathrm{t}, J=7.5,1 \mathrm{H}, \mathrm{SO}_{2} P h\right), 7.66-7.71(\mathrm{~m}, 4 \mathrm{H}$, $\left.\mathrm{SO}_{2} P h\right), 7.75\left(\mathrm{t}, J=7.5,1 \mathrm{H}, \mathrm{SO}_{2} P h\right), 7.90(\mathrm{dd}, J=10.4-2.2,1 \mathrm{H}, H 6), 8.00-8.02\left(\mathrm{~m}, 2 \mathrm{H}, \mathrm{SO}_{2} P h\right), 8.62$ $\left(\mathrm{s}, 1 \mathrm{H}, \mathrm{NHSO} \mathrm{Sh}_{2} \mathrm{Ph}{ }^{13} \mathrm{C}\right.$ NMR $\left(125 \mathrm{MHz}\right.$, acetone-d $\left.\mathrm{d}_{6}\right): 16.15\left(\mathrm{CH}_{3}\right), 37.73(\mathrm{CH}), 44.58(\mathrm{CH}), 51.98$ $(\mathrm{CH}), 68.08(\mathrm{CH}), 92.89(\mathrm{CH}), 111.17(\mathrm{CH}), 121.37(\mathrm{CH}), 124.75(\mathrm{CH}), 127.99(\mathrm{CH}), 128.05(\mathrm{CH})$, $129.77(\mathrm{CH}), 130.09(\mathrm{CH}), 130.66(\mathrm{C}), 131.89(\mathrm{CH}), 132.70(\mathrm{C}), 133.44(\mathrm{CH}), 134.27(\mathrm{CH}), 139.27$ $(\mathrm{CH}), 140.82(\mathrm{C}), 141.53$ (C), 156.89 (C), 176.46 (C), 198.28 (C). N-NMe carbons were not detected, even after processing the FID with a LB value of $35 \mathrm{~Hz}$ : this maybe due to not resolved broad signals for these two non-equivalent carbons. IR (KBr): 3254, 3075, 2944, 2882, 1682, 1615, 1577, 1483, $1448,1317,1202,1162,1150,1088,842,776,730$. HRMS (ESI) calcd for $\mathrm{C}_{30} \mathrm{H}_{31} \mathrm{~N}_{4} \mathrm{O}_{6} \mathrm{~S}_{2}\left(\mathrm{MH}^{+}\right)$ 607.1685, found 607.1687.

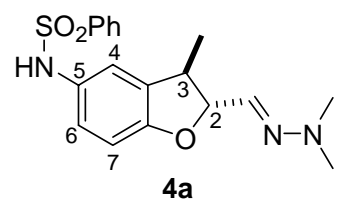

The filtrate was evaporated and purified by flash chromatography (petroleum ether / EtOAc 70:30) to afford 2,3-Dihydrobenzofurane $4 \mathbf{a}$ as a pale yellow solid $(233 \mathrm{mg}, 32 \%)$. Mp $44-45^{\circ} \mathrm{C} .{ }^{1} \mathrm{H}$ NMR (500 $\mathrm{MHz}, \mathrm{CDCl}_{3}$ ): 1.26 (d, $\left.J=7.0,3 \mathrm{H}, \mathrm{CH}_{3}\right) 2.84$ (s, $6 \mathrm{H}, \mathrm{NMe}$ ), 3.38 (m, $\left.1 \mathrm{H}, H 3\right), 4.74$ (dd, $J=8.3-6.6$, $1 \mathrm{H}, H 2), 6.53\left(\mathrm{~d}, J=6.6,1 \mathrm{H}, H \mathrm{C}=\mathrm{N}-\mathrm{NMe}_{2}\right), 6.57(\mathrm{~d}, J=8.4,1 \mathrm{H}, H 7), 6.71(\mathrm{dd}, J=8.4-2.0,1 \mathrm{H}, H 6)$, 6.87 (br s, $1 \mathrm{H}, H 4), 6.91$ (very br s, $1 \mathrm{H}, \mathrm{NHSO}_{2} \mathrm{Ph}$ ), 7.42 (t, $J=7.7,2 \mathrm{H}, \mathrm{SO}_{2}(m-\mathrm{Ar} H)$ ), 7.52 (t, $J=7.6$, $\left.1 \mathrm{H}, \mathrm{SO}_{2}(p-\mathrm{Ar} H)\right), 7.71\left(\mathrm{~d}, J=7.3,2 \mathrm{H}, \mathrm{SO}_{2}(o-\operatorname{Ar} H)\right) .{ }^{13} \mathrm{C} \mathrm{NMR}\left(125 \mathrm{MHz}, \mathrm{CDCl}_{3}\right): 17.74\left(\mathrm{CH}_{3}\right)$, $41.19(\mathrm{CH}), 42.61\left(\mathrm{CH}_{3}\right), 91.66(\mathrm{CH}), 109.67(\mathrm{CH}), 121.00(\mathrm{CH}), 124.81(\mathrm{CH}), 127.46(\mathrm{CH}), 128.84$ (C), $128.98(\mathrm{CH}), 131.12(\mathrm{CH}), 132.89(\mathrm{CH}), 133.49$ (C), 139.01 (C), 157.57 (C). IR (film): 3257, 2962, 2869, 1590, 1483, 1447, 1332, 1204, 1164, 1092, 942, 725, 689, 585, 545. HRMS (EI) calcd for $\mathrm{C}_{18} \mathrm{H}_{21} \mathrm{~N}_{3} \mathrm{O}_{3} \mathrm{~S}\left(\mathrm{M}^{+\bullet}\right)$ 359.1304, found 359.1305. 
Experimental procedure for the tautomerization of compound 5 into 7.

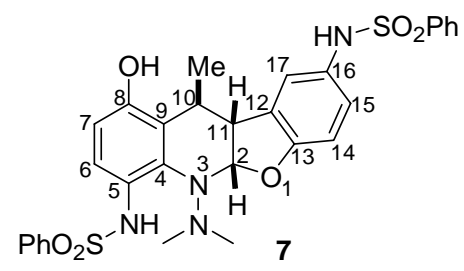

To a solution of compound $5(42.4 \mathrm{mg}, 0.07 \mathrm{mmol})$ in chloroform $(14 \mathrm{~mL})$ was added dropwise at room temperature 1,8-diazabicyclo [5.4.0] undec-7-ene $(11 \mu \mathrm{L}, 0.07 \mathrm{mmol}, 0.5$ equivalent for the two tautomerizable ketone and imine functionalities). The resulting mixture was stirred for 2 hours and the volatiles were then removed under reduced pressure. The residue was purified by flash chromatography (petroleum ether / EtOAc 50:50) to afford tetracyclic compound 7 as a pale pink solid (34.1 mg, 80\%). An analytical sample was obtained by washing this solid with a small volume of chloroform (27.5 mg, 65\%, white solid). Mp $171-172^{\circ} \mathrm{C}$ (decomposed). ${ }^{1} \mathrm{H}$ NMR (500 MHz, acetone$\left.\mathrm{d}_{6}\right): 1.30\left(\mathrm{~d}, J=7.2,3 \mathrm{H}, \mathrm{CH}_{3}\right), 2.71\left(\mathrm{br} \mathrm{s}, 6 \mathrm{H}, \mathrm{NMe} e_{2}\right), 3.65-3.68(\mathrm{~m}, 1 \mathrm{H}, H 10), 3.99(\mathrm{~d}, J=9.2,1 \mathrm{H}$, $H 11), 6.22(\mathrm{~d}, J=9.2,1 \mathrm{H}, H 2), 6.28(\mathrm{~d}, J=8.5,1 \mathrm{H}, H 14), 6.47(\mathrm{~d}, J=8.7,1 \mathrm{H}, H 7), 6.70(\mathrm{dd}, J=$ 8.7-1.6, 1H, H15), $7.02($ br s, $1 \mathrm{H}, H 17), 7.14\left(\mathrm{t}, J=7.8,2 \mathrm{H}, \mathrm{SO}_{2} P h\right), 7.20(\mathrm{~d}, J=8.7,1 \mathrm{H}, H 6), 7.36(\mathrm{t}$, $\left.J=7.8,2 \mathrm{H}, \mathrm{SO}_{2} P h\right), 7.45-7.54\left(\mathrm{~m}, 4 \mathrm{H}, \mathrm{SO}_{2} P h\right), 7.56-7.58\left(\mathrm{~m}, 2 \mathrm{H}, \mathrm{SO}_{2} P h\right), 8.32$ (br s, $\left.1 \mathrm{H}, \mathrm{NHSO}_{2} \mathrm{Ph}\right)$, $8.50\left(\right.$ br s, $\left.1 \mathrm{H}, \mathrm{NHSO}_{2} \mathrm{Ph}\right), 9.98(\mathrm{~s}, 1 \mathrm{H}, \mathrm{ArOH}) .{ }^{13} \mathrm{C}$ NMR $\left(125 \mathrm{MHz}\right.$, acetone-d $\left.\mathrm{d}_{6}\right): 20.18\left(\mathrm{CH}_{3}\right), 27.09$ $(\mathrm{CH}), 43.58\left(\mathrm{CH}_{3}\right), 44.14\left(\mathrm{CH}_{3}\right), 50.43(\mathrm{CH}), 87.70(\mathrm{CH}), 109.24(\mathrm{CH}), 110.62(\mathrm{CH}), 118.95(\mathrm{C})$, $119.35(\mathrm{CH}), 121.17(\mathrm{C}), 121.81(\mathrm{CH}), 123.48(\mathrm{CH}), 127.12(\mathrm{CH}), 127.90(\mathrm{CH}), 129.60(\mathrm{CH}), 129.64$ $(\mathrm{CH}), 130.38(\mathrm{C}), 131.02(\mathrm{C}), 133.35(\mathrm{CH}), 133.37(\mathrm{CH}), 133.78$ (C), $140.43(\mathrm{C}), 141.40(\mathrm{C}), 152.22$ (C), 157.17 (C). The two carbons of $\mathrm{N}-\mathrm{NMe} 2$ consist in two non equivalent very broad signals and were observed after processing the FID with a LB value of $20 \mathrm{~Hz}$. IR (KBr): 3387, 3256, 2972, 1600, $1487,1448,1336,1312,1288,1162,1146,1093,900,806,748,725,685,593,542$. MS (ESI) for $\mathrm{C}_{30} \mathrm{H}_{31} \mathrm{~N}_{4} \mathrm{O}_{6} \mathrm{~S}_{2}\left(\mathrm{MH}^{+}\right): 607$.

The monocrystal for the X-ray diffraction analysis was obtained as follows: a concentrated solution of compound $7(35 \mathrm{mg})$ in acetone- $\mathrm{d}_{6}(500 \mu \mathrm{L})$ was allowed to stand for 5 weeks at $4{ }^{\circ} \mathrm{C}$. A colorless monocrystal $(0.52 \times 0.26 \times 0.24 \mathrm{~mm})$ was analyzed on a Nonius Kappa CCD diffractometer at $293 \mathrm{~K}$.

The crystal structure of compound $\mathbf{7}$ has been deposited at the Cambridge Crystallographic Data Centre (CCDC 604215).

Experimental procedure for the optimization of the $[3+2]$ cycloaddition reaction between quinone monoimide 1a and azadiene $2 a\left(R^{1}=M e\right.$ and $\left.R^{2}=H\right)$.

A solution of quinone monoimide $1 \mathbf{a}(25 \mathrm{mg}, 0.1 \mathrm{mmol})$ in absolute ethanol $(9 \mathrm{~mL})$ was added onto a solution of azadiene $2 \mathbf{a}$ in absolute ethanol $(1 \mathrm{~mL})$ at $0^{\circ} \mathrm{C}$ under argon in one portion or with the help of a syringe pump over the indicated period. After stirring during the indicated time, the volatiles were removed under high vacuum at $0^{\circ} \mathrm{C}$. The residue was analyzed by ${ }^{1} \mathrm{H} N M R$ in $\mathrm{CDCl}_{3}$ at $298 \mathrm{~K}$ : the samples were prepared just before the analysis (a maximum of $5 \mathrm{~min}$ between the preparation and the analysis of the sample was observed). The molar ratio DHBF 4a / tetracyclic compound $\mathbf{5}$ was determined after integration of the doublet of doublet centered at $\delta=4.74 \mathrm{ppm}$ for $\mathbf{4 a}$ and the triplet at $\delta=4.26$ ppm for 5 . 
Typical experimental procedure for the preparation of 5-Sulfonamido-2,3-dihydrobenzofuranes (DHBF): Preparation of compound 4e.

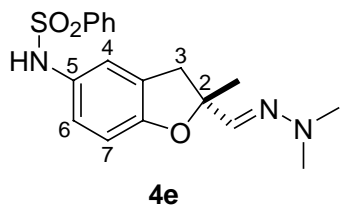

A solution of quinone monoimide $1 \mathbf{a}(495 \mathrm{mg}, 2 \mathrm{mmol})$ in absolute ethanol $(190 \mathrm{~mL})$ was slowly added onto a solution of azadiene $2 \mathbf{f}(1,122 \mathrm{~g}, 10 \mathrm{mmol})$ in absolute ethanol $(10 \mathrm{~mL})$ at $0^{\circ} \mathrm{C}$ under argon with a peristaltic pump over a 5 hours period. After addition, the mixture was stirred for an additional $30 \mathrm{~min}$. After removal of the volatiles under high vacuum at $23^{\circ} \mathrm{C}$, the residue was purified by flash chromatography (petroleum ether / EtOAc 70:30) to afford DHBF 4e (pale orange very viscous oil, $669 \mathrm{mg}, 93 \%) .{ }^{1} \mathrm{H} \mathrm{NMR}\left(300 \mathrm{MHz}, \mathrm{CDCl}_{3}\right): 1.54$ (s, 3H, $\left.\mathrm{CH}_{3}\right), 2.77$ (s, 6H, NMe $), 2.88$ (d, $J=16.1,1 \mathrm{H}, H 3), 3.56$ (d, $J=16.1,1 \mathrm{H}, H 3$ '), 6.52 (d, $J=8.4,1 \mathrm{H}, H 7), 6.61$ (s, $1 \mathrm{H}, H \mathrm{C}=\mathrm{N}-\mathrm{NMe}_{2}$ ), 6.68 (dd, $J=8.4-2.3,1 \mathrm{H}, H 6$ ), 6.95 (br d, $J=2.3,1 \mathrm{H}, H 4), 7.09$ (br s, $1 \mathrm{H}, \mathrm{NHSO}_{2} \mathrm{Ph}$ ), 7.41 (t, $J=7.4$, $\left.2 \mathrm{H}, \mathrm{SO}_{2}(m-\mathrm{Ar} H)\right), 7.51\left(\mathrm{t}, J=7.4,1 \mathrm{H}, \mathrm{SO}_{2}(p-\mathrm{ArH})\right), 7.71-7.75\left(\mathrm{~m}, 2 \mathrm{H}, \mathrm{SO}_{2}(o-\operatorname{Ar} H)\right) .{ }^{13} \mathrm{C}$ NMR $(75$ $\left.\mathrm{MHz}, \mathrm{CDCl}_{3}\right): 25.65\left(\mathrm{CH}_{3}\right), 39.70\left(\mathrm{CH}_{2}\right), 42.76\left(\mathrm{CH}_{3}\right), 89.24(\mathrm{C}), 109.52(\mathrm{CH}), 122.10(\mathrm{CH}), 124.39$ $(\mathrm{CH}), 127.39(\mathrm{CH}), 128.43(\mathrm{C}), 128.49(\mathrm{C}), 128.95(\mathrm{CH}), 132.83(\mathrm{CH}), 135.74(\mathrm{CH}), 139.05(\mathrm{C})$, 157.00 (C). IR (film): 3262, 3066, 2967, 2928, 2859, 2790, 1601, 1487, 1447, 1330, 1250, 1162, 1092, $1029,892,755,724,689$. HRMS (EI) calcd for $\mathrm{C}_{18} \mathrm{H}_{21} \mathrm{~N}_{3} \mathrm{O}_{3} \mathrm{~S}\left(\mathrm{M}^{+\bullet}\right) 359.1304$, found 359.1303.

\section{Compound 4b.}

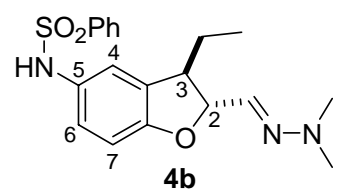

Purified by flash chromatography (petroleum ether / EtOAc 60:40) to afford DHBF $\mathbf{4 b}$ as a pale green very viscous oil, 53\%. ${ }^{1} \mathrm{H}$ NMR (300 MHz, $\left.\mathrm{CDCl}_{3}\right)$ : $0.88\left(\mathrm{t}, J=7.4,3 \mathrm{H}, \mathrm{CH}_{2} \mathrm{CH}_{3}\right), 1.57-1.76(\mathrm{~m}, 2 \mathrm{H}$, $\left.\mathrm{CH}_{2} \mathrm{CH}_{3}\right), 2.82\left(\mathrm{~s}, 6 \mathrm{H}, \mathrm{NMe} e_{2}\right), 3.29(\mathrm{q}, J=6.5,1 \mathrm{H}, H 3), 4.90(\mathrm{t}, J=6.5,1 \mathrm{H}, H 2), 6.50(\mathrm{~d}, J=6.5,1 \mathrm{H}$, $\left.H \mathrm{C}=\mathrm{N}-\mathrm{NMe}_{2}\right), 6.58(\mathrm{~d}, J=8.6,1 \mathrm{H}, H 7), 6.72(\mathrm{dd}, J=8.6-2.2,1 \mathrm{H}, H 6), 6.80\left(\right.$ br s, $\left.1 \mathrm{H}, \mathrm{NHSO}_{2} \mathrm{Ph}\right)$, $6.88(\mathrm{~d}, J=2.2,1 \mathrm{H}, H 4), 7.42\left(\mathrm{t}, J=7.5,2 \mathrm{H}, \mathrm{SO}_{2}(m-\mathrm{Ar} H)\right), 7.53\left(\mathrm{t}, J=7.5,1 \mathrm{H}, \mathrm{SO}_{2}(p-\mathrm{Ar} H)\right), 7.69-$ $7.72\left(\mathrm{~m}, 2 \mathrm{H}, \mathrm{SO}_{2}(o-\mathrm{ArH})\right) .{ }^{13} \mathrm{C} \mathrm{NMR}\left(75 \mathrm{MHz}, \mathrm{CDCl}_{3}\right): 10.94\left(\mathrm{CH}_{3}\right), 26.61\left(\mathrm{CH}_{2}\right), 42.64\left(\mathrm{CH}_{3}\right), 47.52$ $(\mathrm{CH}), 89.42(\mathrm{CH}), 109.66(\mathrm{CH}), 121.62(\mathrm{CH}), 125.04(\mathrm{CH}), 127.47(\mathrm{CH}), 128.59(\mathrm{C}), 128.99(\mathrm{CH})$, 131.88 (C), $132.05(\mathrm{CH}), 132.91(\mathrm{CH}), 138.99$ (C), 157.86 (C). IR (film): 3261, 3066, 2963, 2929, 2876, 1588, 1486, 1447, 1403, 1331, 1242, 1204, 1161, 1092, 962, 912, 824, 755, 726, 689. HRMS (EI) calcd for $\mathrm{C}_{19} \mathrm{H}_{23} \mathrm{~N}_{3} \mathrm{O}_{3} \mathrm{~S}\left(\mathrm{M}^{+\bullet}\right)$ 373.1460, found 373.1463 .

\section{Compound 4d.}

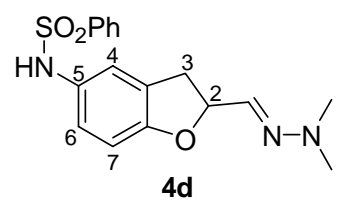

Purified by flash chromatography (petroleum ether / EtOAc 60:40) to afford DHBF 4d as a pale yellow very viscous oil, $73 \%$. ${ }^{1} \mathrm{H}$ NMR (300 $\mathrm{MHz}, \mathrm{CDCl}_{3}$ ): 2.84 (s, $6 \mathrm{H}, \mathrm{NMe}$ ), 3.15-3.23 (m, 1H, H3), 
3.27-3.36 (m, 1H, H3'), 5.27-5.35 (m, $1 \mathrm{H}, H 2), 6.47$ (br s, $\left.1 \mathrm{H}, \mathrm{NHSO}_{2} \mathrm{Ph}\right), 6.54-6.59(\mathrm{~m}, 2 \mathrm{H}, H \mathrm{C}=\mathrm{N}-$ $\left.\mathrm{NMe}_{2} \& H 7\right), 6.64$ (dd, $\left.J=8.7-2.3,1 \mathrm{H}, H 6\right), 6.98$ (br s, $\left.1 \mathrm{H}, H 4\right), 7.44\left(\mathrm{t}, J=7.2,2 \mathrm{H}, \mathrm{SO}_{2}(m-\mathrm{Ar} H)\right.$ ), $7.54\left(\mathrm{t}, J=7.2,1 \mathrm{H}, \mathrm{SO}_{2}(p-\mathrm{ArH})\right), 7.69-7.73\left(\mathrm{~m}, 2 \mathrm{H}, \mathrm{SO}_{2}(o-\mathrm{Ar} H)\right) .{ }^{13} \mathrm{C} \mathrm{NMR}\left(75 \mathrm{MHz}, \mathrm{CDCl}_{3}\right): 34.23$ $\left(\mathrm{CH}_{2}\right), 42.60\left(\mathrm{CH}_{3}\right), 83.87(\mathrm{CH}), 109.47(\mathrm{CH}), 121.96(\mathrm{CH}), 124.52(\mathrm{CH}), 127.39(\mathrm{CH}), 128.15(\mathrm{C})$, $128.70(\mathrm{C}), 129.01(\mathrm{CH}), 131.83(\mathrm{CH}), 132.91(\mathrm{CH}), 139.04(\mathrm{C}), 157.90$ (C). IR (film): 3262, 2957, 2860, 2792, 1661, 1594, 1487, 1447, 1331, 1230, 1207, 1161, 1092, 1050, 954, 828, 755, 725, 689. HRMS (EI) calcd for $\mathrm{C}_{17} \mathrm{H}_{19} \mathrm{~N}_{3} \mathrm{O}_{3} \mathrm{~S}\left(\mathrm{M}^{+\bullet}\right) 345.1147$, found 345.1149 .

\section{Compound 4f.}

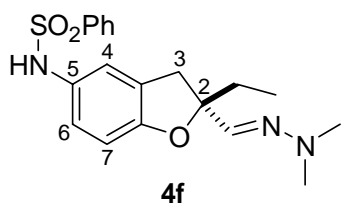

Purified by flash chromatography (petroleum ether / EtOAc 60:40) to afford DHBF $\mathbf{4 f}$ as a pale yellow solid, 90\%. Mp 114-115 ${ }^{\circ} \mathrm{C}$. ${ }^{1} \mathrm{H}$ NMR (300 MHz, $\mathrm{CDCl}_{3}$ ): 0.91 (t, $\left.J=7.4,3 \mathrm{H}, \mathrm{CH}_{2} \mathrm{CH}_{3}\right), 1.79-1.96$ (m, $\left.2 \mathrm{H}, \mathrm{CH}_{2} \mathrm{CH}_{3}\right), 2.78\left(\mathrm{~s}, 6 \mathrm{H}, \mathrm{NMe}\right.$ ) $2.92(\mathrm{~d}, J=15.8,1 \mathrm{H}, H 3), 3.64\left(\mathrm{~d}, J=15.8,1 \mathrm{H}, H 3{ }^{\prime}\right), 6.26(\mathrm{~s}, 1 \mathrm{H}$, $\left.\mathrm{NHSO}_{2} \mathrm{Ph}\right), 6.53-6.56\left(\mathrm{~m}, 2 \mathrm{H}, H \mathrm{C}=\mathrm{N}-\mathrm{NMe}_{2} \& H 7\right), 6.62(\mathrm{dd}, J=8.7-2.3,1 \mathrm{H}, H 6), 6.92(\mathrm{br} \mathrm{s}, 1 \mathrm{H}, H 4)$, 7.41-7.46 (m, 2H, SO $(m-\mathrm{ArH})), 7.54\left(\mathrm{t}, J=7.5,1 \mathrm{H}, \mathrm{SO}_{2}(p-\mathrm{Ar} H)\right), 7.68-7.71\left(\mathrm{~m}, 2 \mathrm{H}, \mathrm{SO}_{2}(o-\operatorname{Ar} H)\right)$. ${ }^{13} \mathrm{C}$ NMR $(75 \mathrm{MHz}, \mathrm{CDCl} 3): 8.05\left(\mathrm{CH}_{3}\right), 32.15\left(\mathrm{CH}_{2}\right), 36.53\left(\mathrm{CH}_{2}\right), 42.87\left(\mathrm{CH}_{3}\right), 92.03(\mathrm{C}), 109.26$ $(\mathrm{CH}), 122.07(\mathrm{CH}), 124.35(\mathrm{CH}), 127.41(\mathrm{CH}), 128.35(\mathrm{C}), 128.65(\mathrm{C}), 128.95(\mathrm{CH}), 132.83(\mathrm{CH})$, $135.52(\mathrm{CH}), 139.09$ (C), 157.28 (C). IR (KBr): 3234, 2972, 2931, 2904, 2865, 1597, 1483, 1467, 1447, 1405, 1329, 1160, 1090, 959, 906, 812, 757, 725, 688, 583, 549. HRMS (EI) calcd for $\mathrm{C}_{19} \mathrm{H}_{23} \mathrm{~N}_{3} \mathrm{O}_{3} \mathrm{~S}\left(\mathrm{M}^{+\bullet}\right) 373.1460$, found 373.1461 .

\section{Compound 8.}

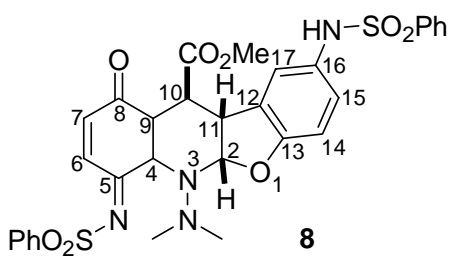

Purified by flash chromatography (petroleum ether / EtOAc 60:40) to afford tetracyclic compound $\mathbf{8}$ as a dark green solid, $32 \%$. Mp 89-91 ${ }^{\circ} \mathrm{C}$ (decomposed). ${ }^{1} \mathrm{H}$ NMR $\left(300 \mathrm{MHz}, \mathrm{CDCl}_{3}\right): 2.21$ (dd, $J=10.6-$ $4.4,1 \mathrm{H}, H 10$ ), 2.36 (br s, 6H, NMe $), 3.45$ (dd, $J=10.6-5.9,1 \mathrm{H}, H 11$ ), 3.59 (dd, $J=4.4-1.9,1 \mathrm{H}, H 9$ ), $3.68\left(\mathrm{~s}, 3 \mathrm{H}, \mathrm{CO}_{2} M e\right), 4.40$ (br t, $\left.J=1.9,1 \mathrm{H}, H 4\right), 5.55(\mathrm{~d}, J=5.9,1 \mathrm{H}, H 2), 6.22\left(\mathrm{br} \mathrm{s}, 1 \mathrm{H}, \mathrm{NHSO}_{2} \mathrm{Ph}\right.$ ), $6.48(\mathrm{~d}, J=10.6,1 \mathrm{H}, H 7), 6.62(\mathrm{~d}, J=8.7,1 \mathrm{H}, H 14), 6.98$ (dd, $J=8.7-2.3,1 \mathrm{H}, H 15), 7.05$ (d, $J=2.3$, $1 \mathrm{H}, H 17$ ), 7.40 (t, $\left.J=7.5,2 \mathrm{H}, \mathrm{SO}_{2} P h\right), 7.49-7.59$ (m, 3H, $\left.\mathrm{H6} \& \mathrm{SO}_{2} P h\right), 7.63-7.68$ (m, 3H, $\left.\mathrm{SO}_{2} P h\right)$, 7.99-8.02 (m, 3H, SO $\left.{ }_{2} P h\right) .{ }^{13} \mathrm{C}$ NMR $\left(75 \mathrm{MHz}\right.$, acetone- $\left.\mathrm{d}_{6}\right): 39.32(\mathrm{CH}), 40.87\left(\mathrm{CH}_{3}\right), 43.35\left(\mathrm{CH}_{3}\right)$, $45.16(\mathrm{CH}), 50.21\left(\mathrm{CH}_{3}\right), 52.18(\mathrm{CH}), 65.83(\mathrm{CH}), 91.83(\mathrm{CH}), 111.00(\mathrm{CH}), 122.37(\mathrm{CH}), 124.68$ $(\mathrm{CH}), 127.94(\mathrm{CH}), 128.09(\mathrm{CH}), 129.61(\mathrm{CH}), 130.09(\mathrm{CH}), 131.11(\mathrm{C}), 132.52(\mathrm{C}), 133.37(\mathrm{CH})$, $133.44(\mathrm{CH}), 134.34(\mathrm{CH}), 137.25(\mathrm{CH}), 140.68(\mathrm{C}), 141.33(\mathrm{C}), 156.60(\mathrm{C}), 171.96(\mathrm{C}), 175.33(\mathrm{C})$, 196.40 (C). The two carbons of $\mathrm{N}-\mathrm{NMe} e_{2}$ consist in two non equivalent broad signals and were observed after processing the FID with a LB value of $20 \mathrm{~Hz}$. IR (KBr): 3262, 3066, 2952, 2924, 2859, 1741, 1692, 1620, 1579, 1481, 1448, 1328, 1239, 1200, 1163, 1090, 825, 783, 757, 727, 689, 595, 549. HRMS (ESI) calcd for $\mathrm{C}_{31} \mathrm{H}_{31} \mathrm{~N}_{4} \mathrm{O}_{8} \mathrm{~S}_{2}\left(\mathrm{MH}^{+}\right)$651.1583, found 651.1585. 
$9 S E Z Z^{\circ}$

IOSZ'I

IOOt'I

$t S \mathcal{E} t^{\circ} I$

$\varepsilon I \angle t^{\circ} I$

$0050^{\circ} z$

9eze 2

$+S \mathcal{E} 8^{\circ} Z$

$6958^{\circ} \mathrm{Z}$

$5698^{\circ} \mathrm{Z}$

$96 \angle 8^{\circ} Z$

$\$ 6 \angle 0^{\circ} \mathcal{E}$

$\varepsilon 9 \varsigma Z^{\circ} t$

$0 \angle 99^{\circ} \mathrm{S}$

$I \angle \angle 9^{\circ} S$

$8605^{\circ} 9$

$90 \varepsilon 5.9$

ZE $19^{\circ} 9$

ZOE ${ }^{\circ} 9$

EE $16^{\circ} 9$

$\angle \angle I 6^{\circ} 9$

EOE $6^{\circ} 9$

$\angle \supset \mathcal{E} 6^{\circ} 9-$

$9 S+0^{\circ} \mathrm{L}$

$+6+0^{\circ} \mathrm{L}$

$8880^{\circ} \mathrm{L}-$

$\mathcal{E O O S} \angle$

O6IS $\angle$

OZLS $\angle-=$

I $\angle 8 S^{\circ} \mathrm{L}$

$9109^{\circ} \mathrm{L}$

$0+99^{\circ} \mathrm{L}$

$S 8 \angle 9^{\circ} \mathrm{L}$

$6+69^{\circ} \mathrm{L}$

$6 I I L{ }^{\circ} \mathrm{L}$

$6 S E L^{\circ} L$

$O I S L^{\circ} \angle$

$S S 9 \angle{ }^{\circ} \mathrm{L}$

$\angle 688^{\circ} \mathrm{L}$

$S E 68^{\circ} \mathrm{L}$

I I I6 $6^{\circ} \mathrm{L}$

$\varepsilon+16^{\circ} \mathrm{L}$

$\varepsilon 900^{\circ} 8$

tIZ0 8

$8229^{\circ} 8$
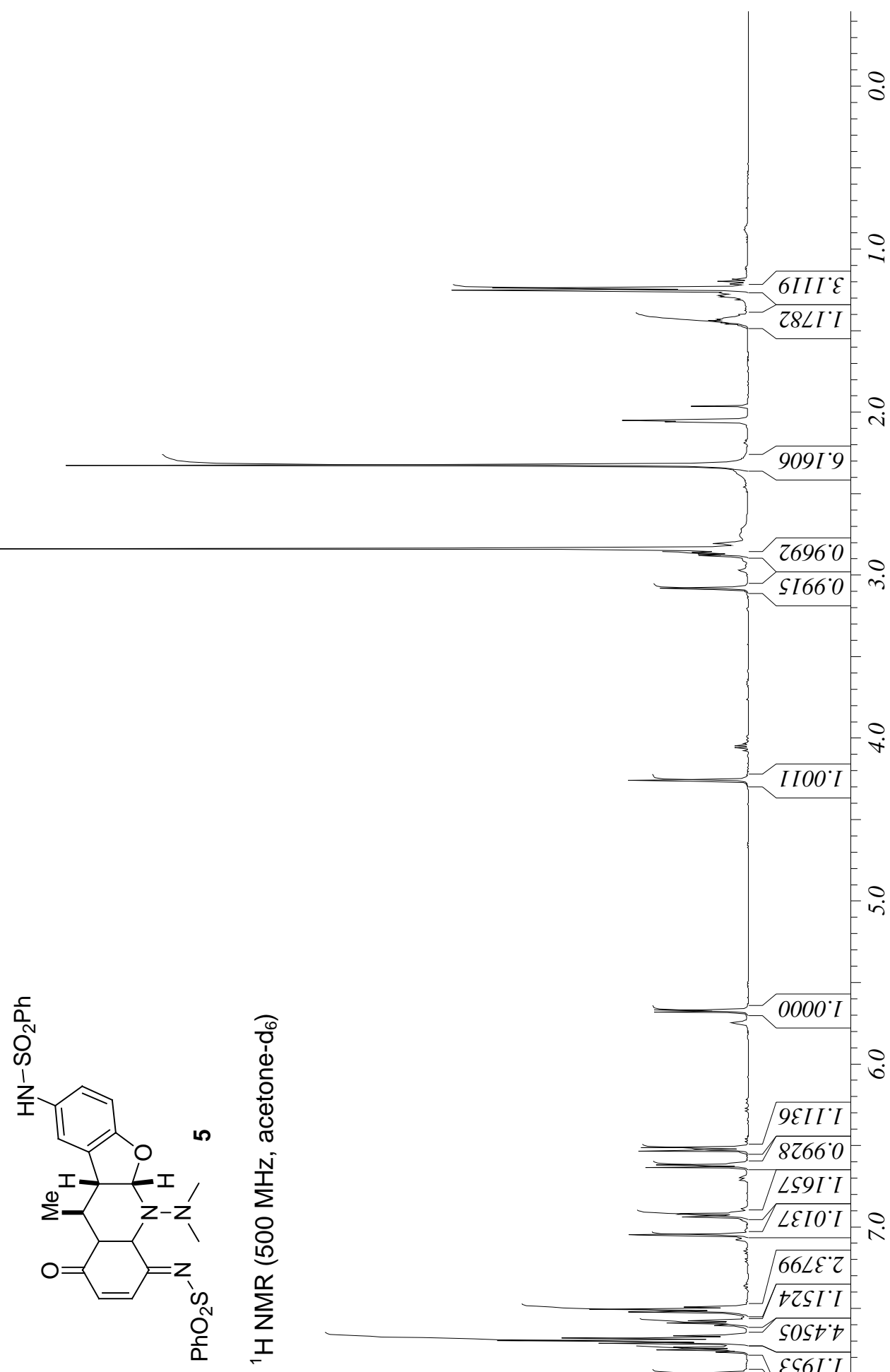


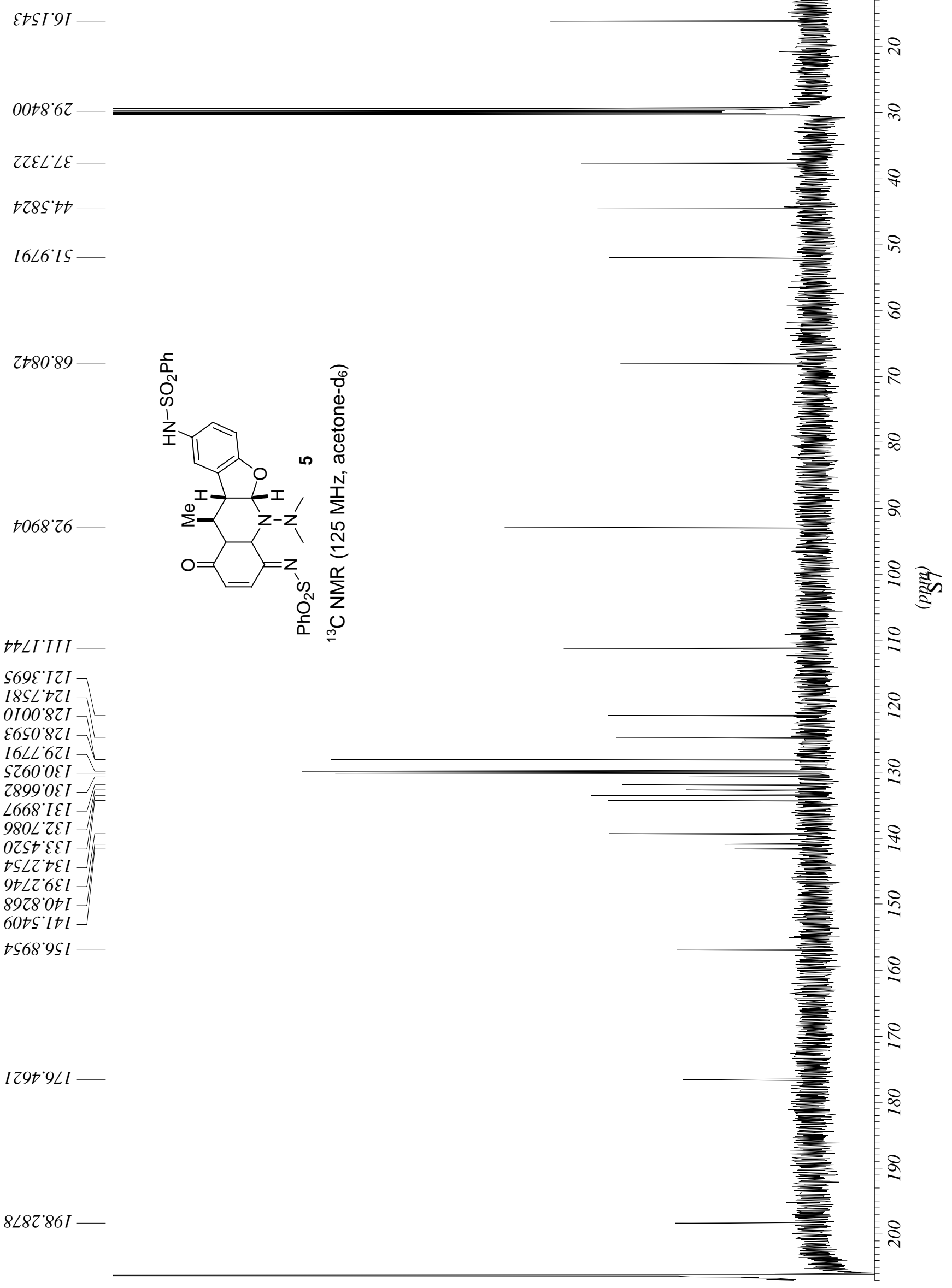


$\varepsilon t S I^{\circ} 9 I$

$6+2 \angle L^{\circ} \angle E$

$I S \angle S+t$

I6L6 IS

$69 \angle 0^{\circ} 89$

t068'26

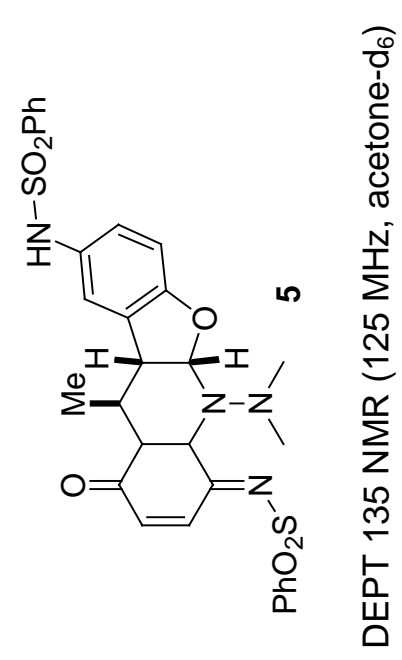

$t D L I^{\circ} I I I-$

S69E'IZI

I8SL $\supset Z I$

$01000^{\circ} 8 Z I$
E6S0

I6LL'6ZI-F

SZ60 $0 \mathcal{\circ} I$

$\angle 668^{\circ} \mathrm{LEI}$

Z6St $\mathcal{E} \mathcal{E} I$

$\angle Z 8 Z^{\circ} \bullet \mathcal{E} I$

$9+\angle Z^{\circ} 6 \varepsilon I$

8

尺

$\infty$

ฉ

8

$=0$

=

$\stackrel{ }{7}$

$\stackrel{n}{-}$

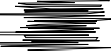

놀
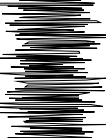

-

8

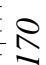

$\infty$

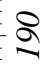


$6 \angle 87^{\circ} I$

๑๐OE' $I$

$00 \varsigma 0 \cdot 2$

$\$ 60 L^{\circ} \mathrm{Z}$

${ }^{8 \varepsilon \mathcal{E} 6} \mathrm{Z}$

$\pitchfork \subseteq \mathcal{E} 9^{\circ} \mathcal{E}$

$\varepsilon \angle t 9^{\circ} \mathcal{E}$

$66 \neq 9^{\circ} \mathcal{E}$

$8199^{\circ} \mathcal{E}$

$t \$ 99^{\circ} \mathcal{E}$

$\varepsilon 9 \angle 9^{\circ} \mathrm{E}$

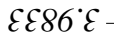

$9100^{\circ}$

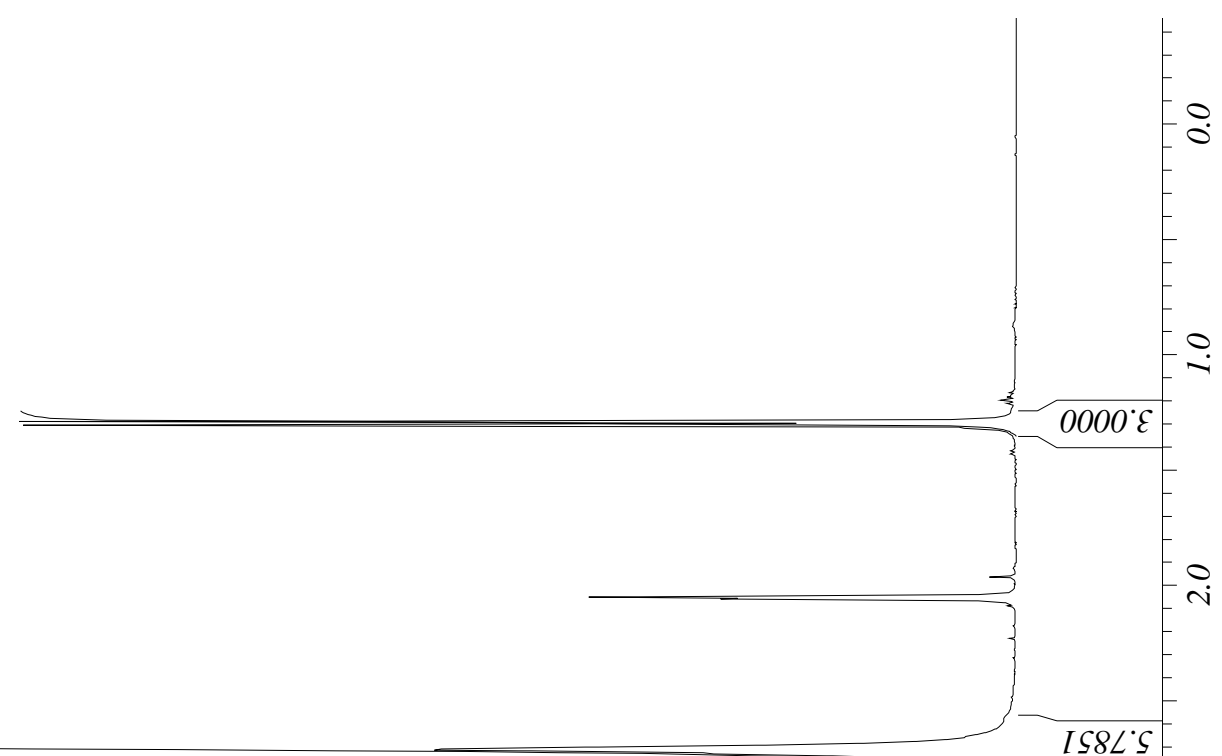

ᄂ

t0I $2^{\circ} 9$

E627'9

$\angle 692^{\circ} 9$

$\angle 982^{\circ} 9$

[8St 9

ZS $\angle t^{\circ} 9$

0269.9

St69.9

t80L' 9

SII $\angle{ }^{\circ} 9$

$8+10^{\circ} \mathrm{L}$

$282 I^{\circ} \mathrm{L}$

$0 t+I^{\circ} L$

$\angle 6 S I^{\circ} \mathrm{L}$

S26I'L

ZOIZ $\angle$

$\mathcal{E} I t E \angle$

$O \angle S E \angle$

$Z Z \angle E \angle$

$\angle t t t^{\circ} L$

Z6St $L$

$\varepsilon+\angle t^{\circ} L$

E98t' $L$

$8005^{\circ} \mathrm{L}$

ZOIS L

ESZS $L$

SOt $S^{\circ} \mathrm{L}$

O09S'L

$S+\angle S^{\circ} \angle$

$\angle \angle I E E^{\circ} 8$

$\angle 96 t^{\circ} 8$

$0086^{\circ} 6$

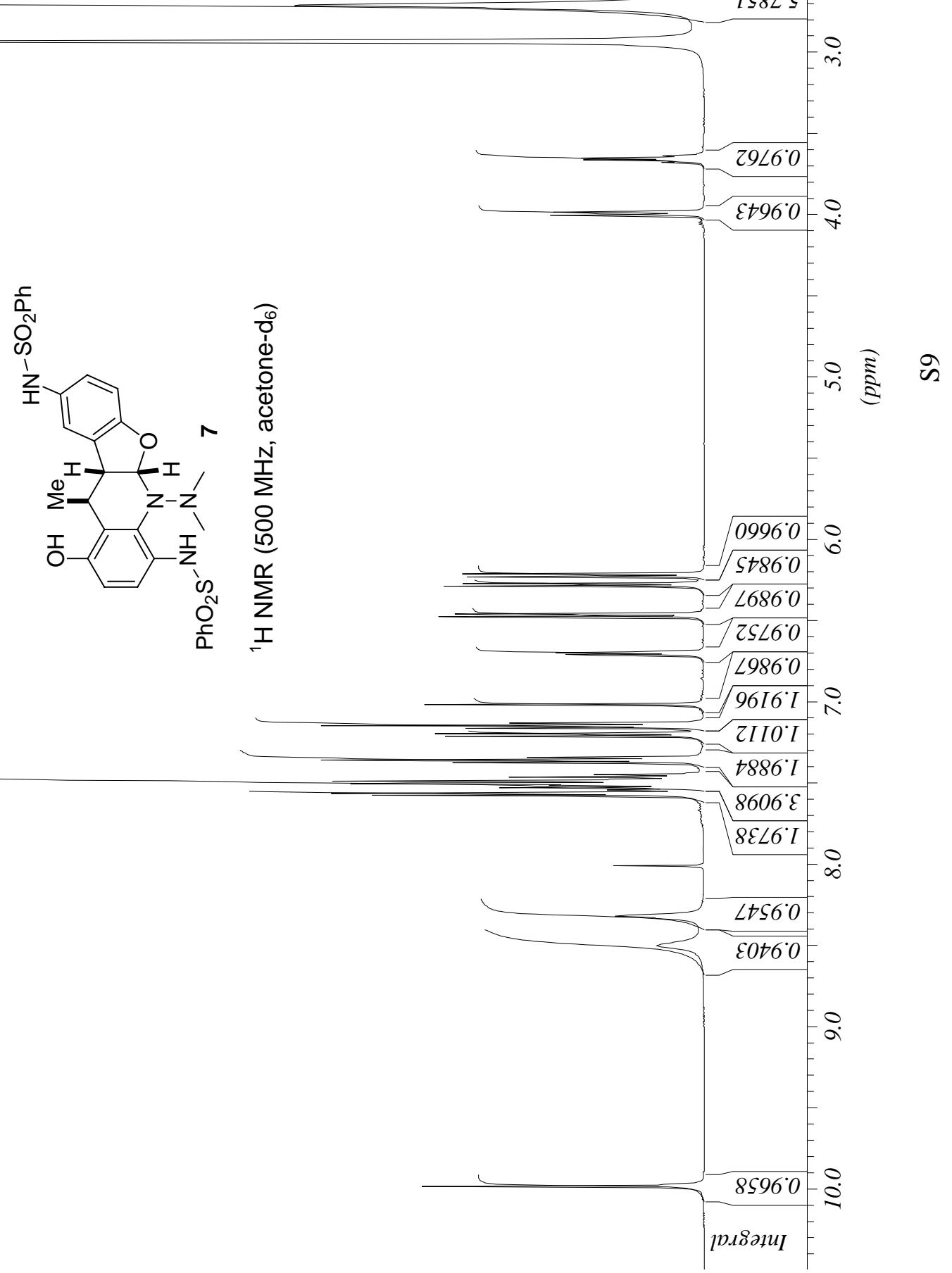




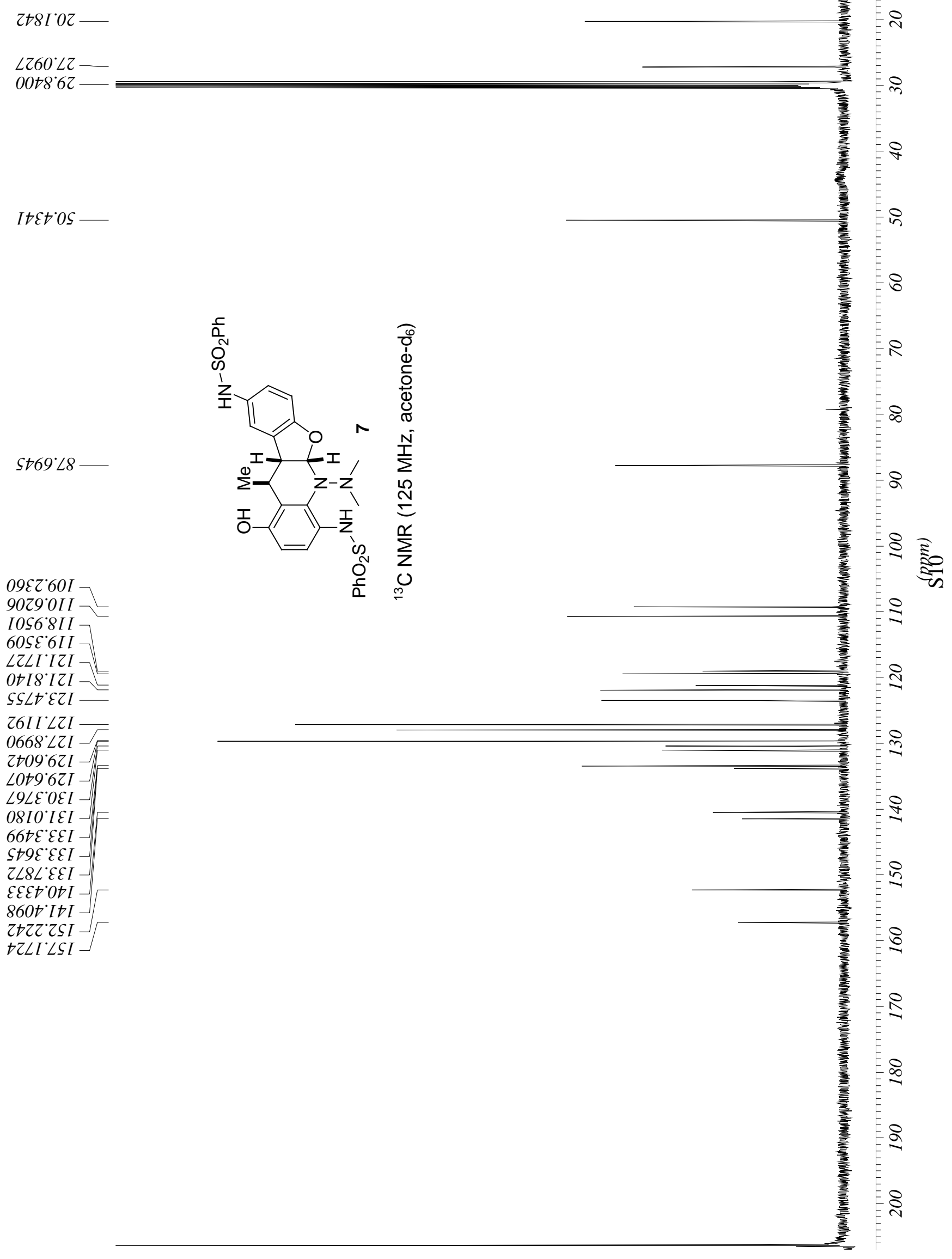



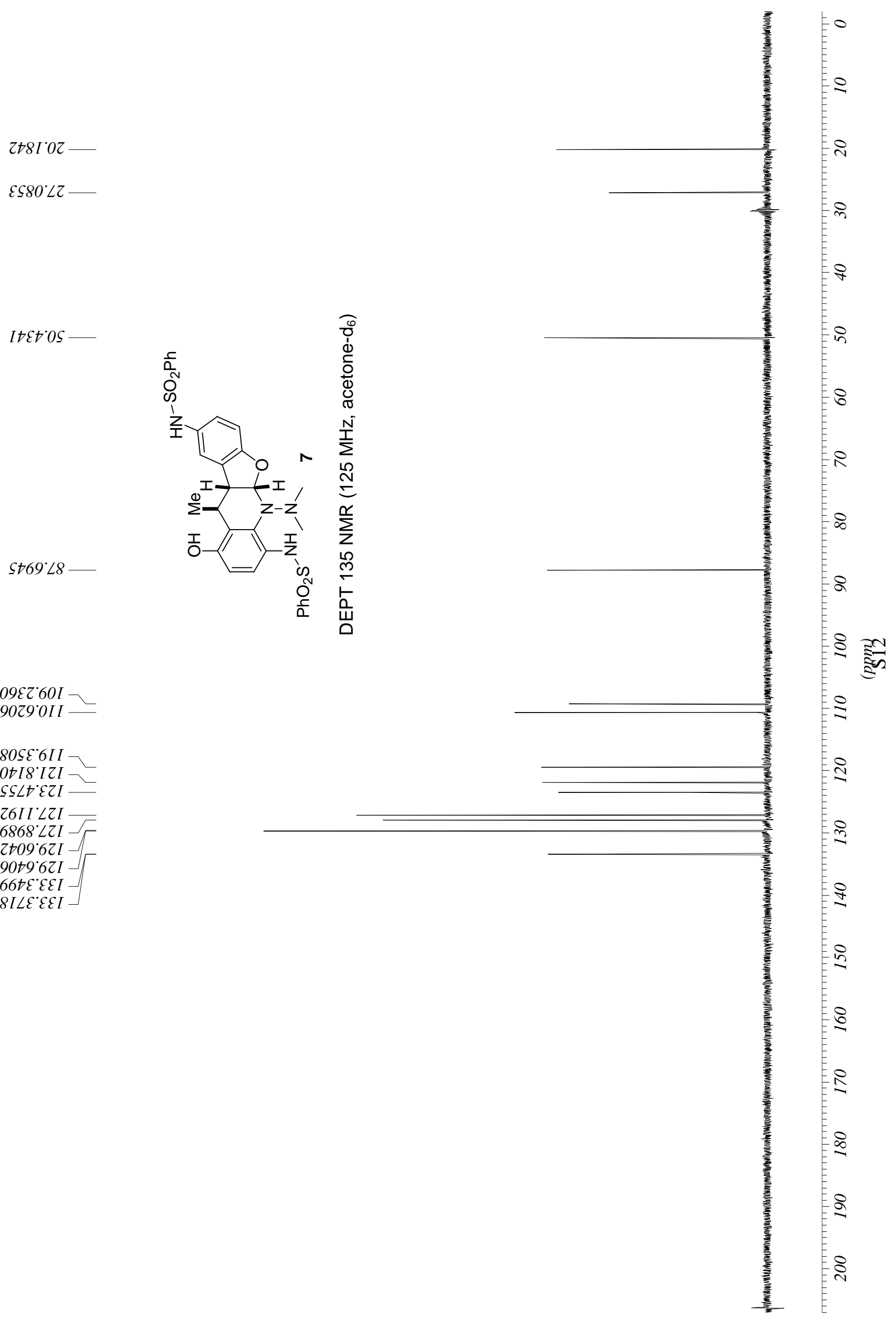
S8ZL't IIt L ' $8+t \angle{ }^{\circ} t$ ISSL't

$8125^{\circ} 9$ $S+\varepsilon \varsigma^{\circ} 9$ It $9 S^{\circ} 9$ $\cos ^{\circ} 9=$

$\angle \angle 69^{\circ} 9$ $600<9$

$I+I \angle{ }^{\circ}$ $6 \angle I \angle{ }^{\circ} 9$ $5898^{\circ} 9$

t0L8 9

tII $16^{\circ} 9$

$0092^{\circ} \mathrm{L}$

$900 t^{\circ} \mathrm{L}$ $\angle S I D^{\circ} \mathrm{L}$ $S I E T^{\circ} \mathrm{L}$ $8 S O S^{\circ} \mathrm{L}$ OIZS'L I9ES $\mathrm{L}$ $8 \mathcal{E} O L^{\circ} \mathrm{L}$ $\varepsilon 90 \angle \mathrm{L}$ $802 L L$

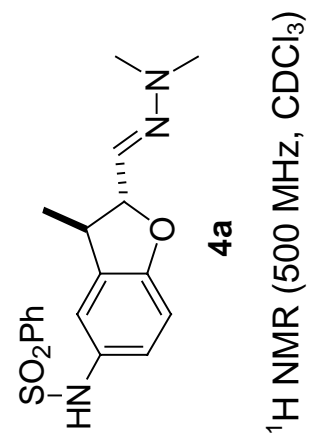

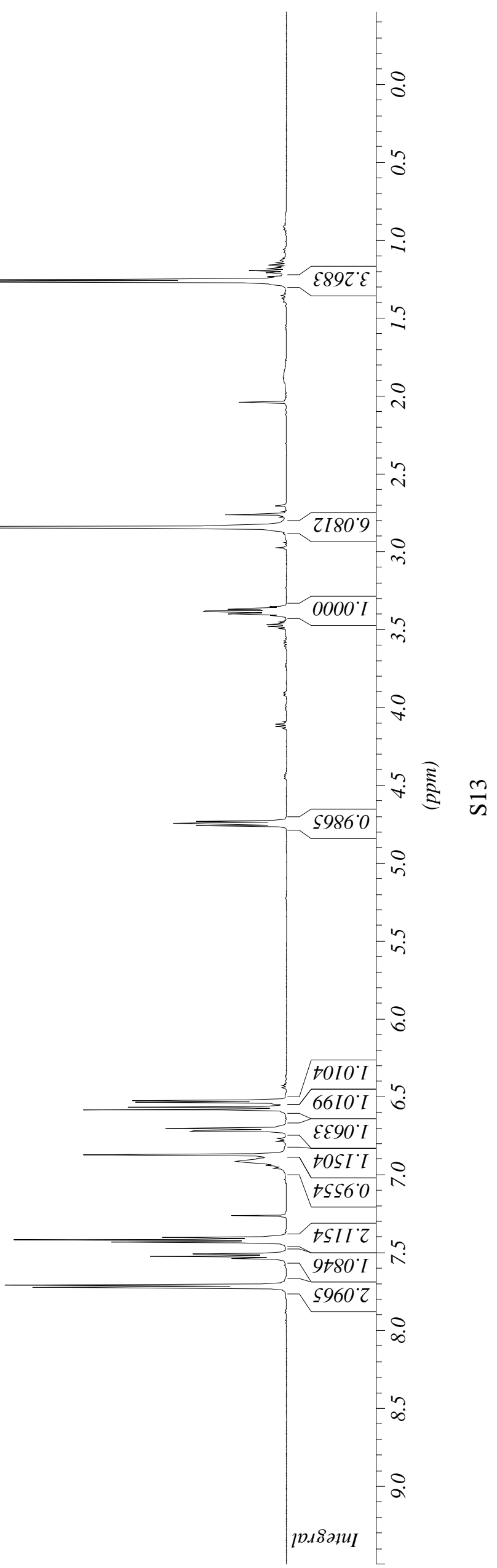


$88 \mathcal{L} L \angle I$

Ez8I'It

9019'2t—

O09I'LL

$6199^{\circ} 16$

0699601

$8000^{\circ}$ IZI

IZI8'tZI -

$S \angle S+\angle Z I$

IZ\$8'8ZI -

S086 $82 I-F$

OEZI'IEI -

$8 \mathcal{8} 68^{\circ} \mathrm{ZEI}$

$+I 6 t^{\circ} \varepsilon \mathcal{E} I-$

ZSIO $6 E I-$

$689 S^{\circ} \angle S I$

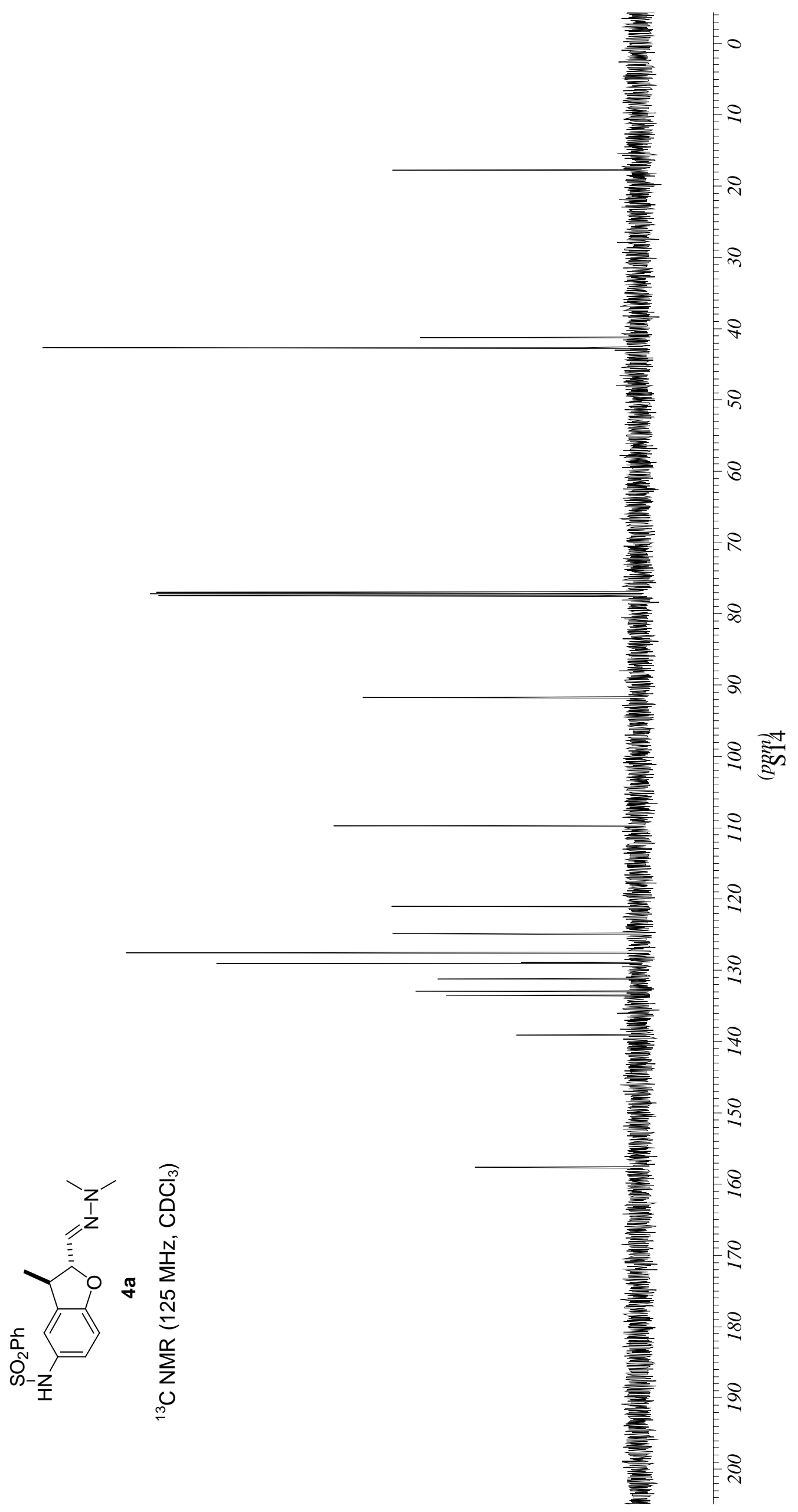




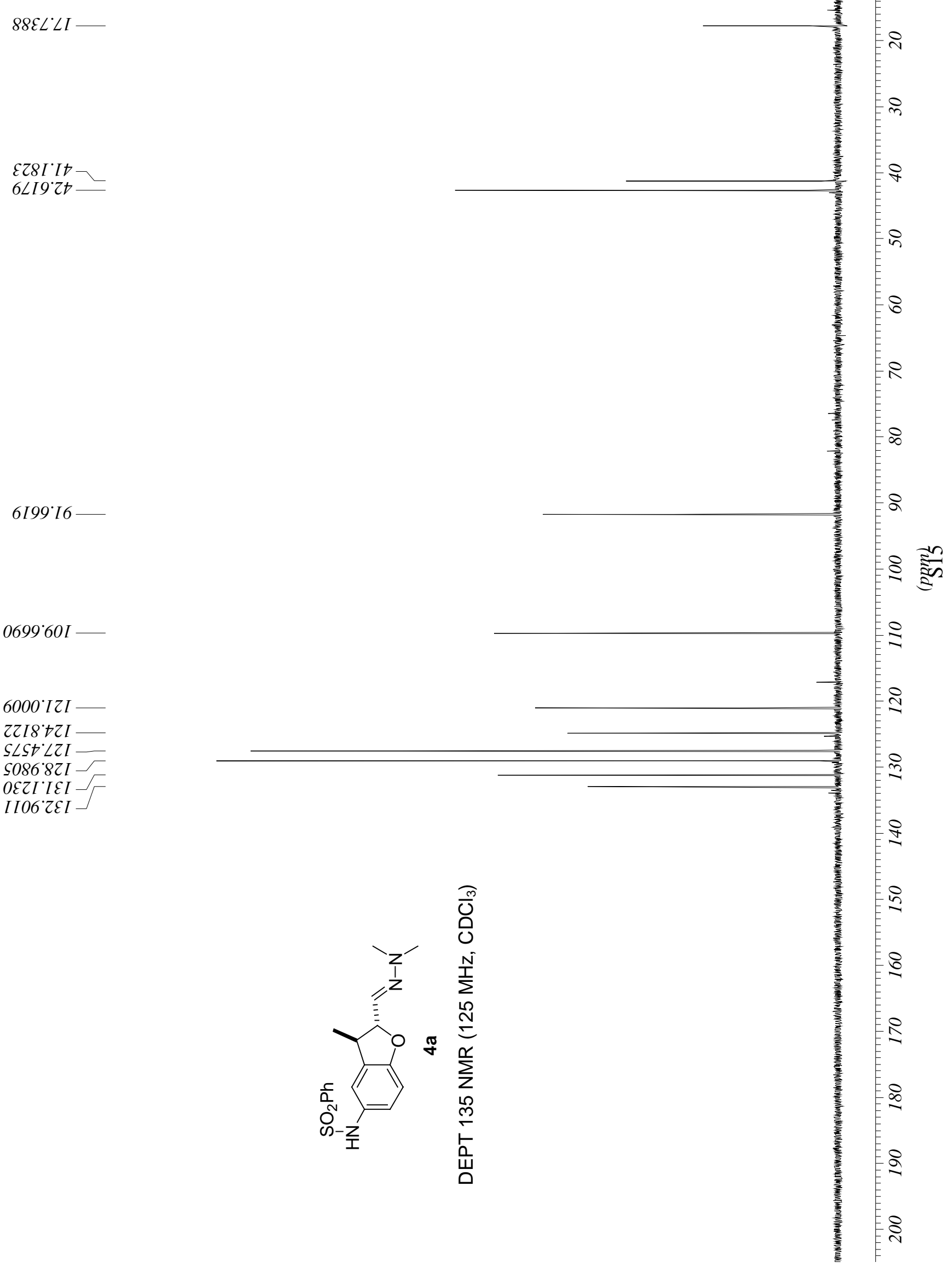




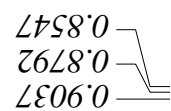

$\mathcal{E S} \angle S^{\circ} I$

$86 S L^{\circ} I$

S088't

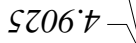

st26.t

$6+6 t^{\circ} 9$

29IS 9

ZS9S. 9

$+\varepsilon 65^{\circ} 9$

$S+0 \angle{ }^{\circ} 9$

80I $\angle$ '

IZE $\angle$ ' 9

$\leftarrow 8 \mathcal{E} \angle L^{\circ} 9$

$9008^{\circ} 9$

$S 188^{\circ} 9$

$6588^{\circ} 9-{ }^{\circ}$

$0092^{\circ} \mathrm{L}$

$\angle E 6 \varepsilon^{\circ} \angle$

$S \angle I D^{\circ} L$

EEtt $L$

9IOS' $\mathrm{L}$

I92S' $\mathrm{L}$

$90 S S^{\circ} \mathrm{L}$

IE69 L

$I 869^{\circ} \mathrm{L}$

$O Z Z L L$

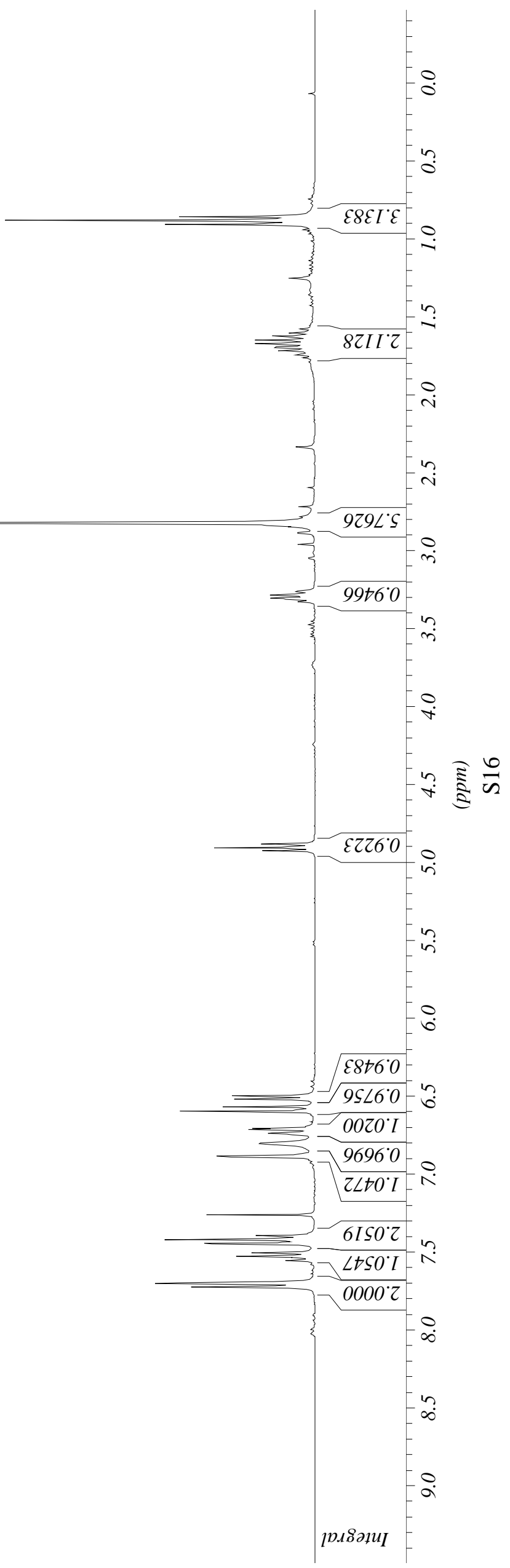




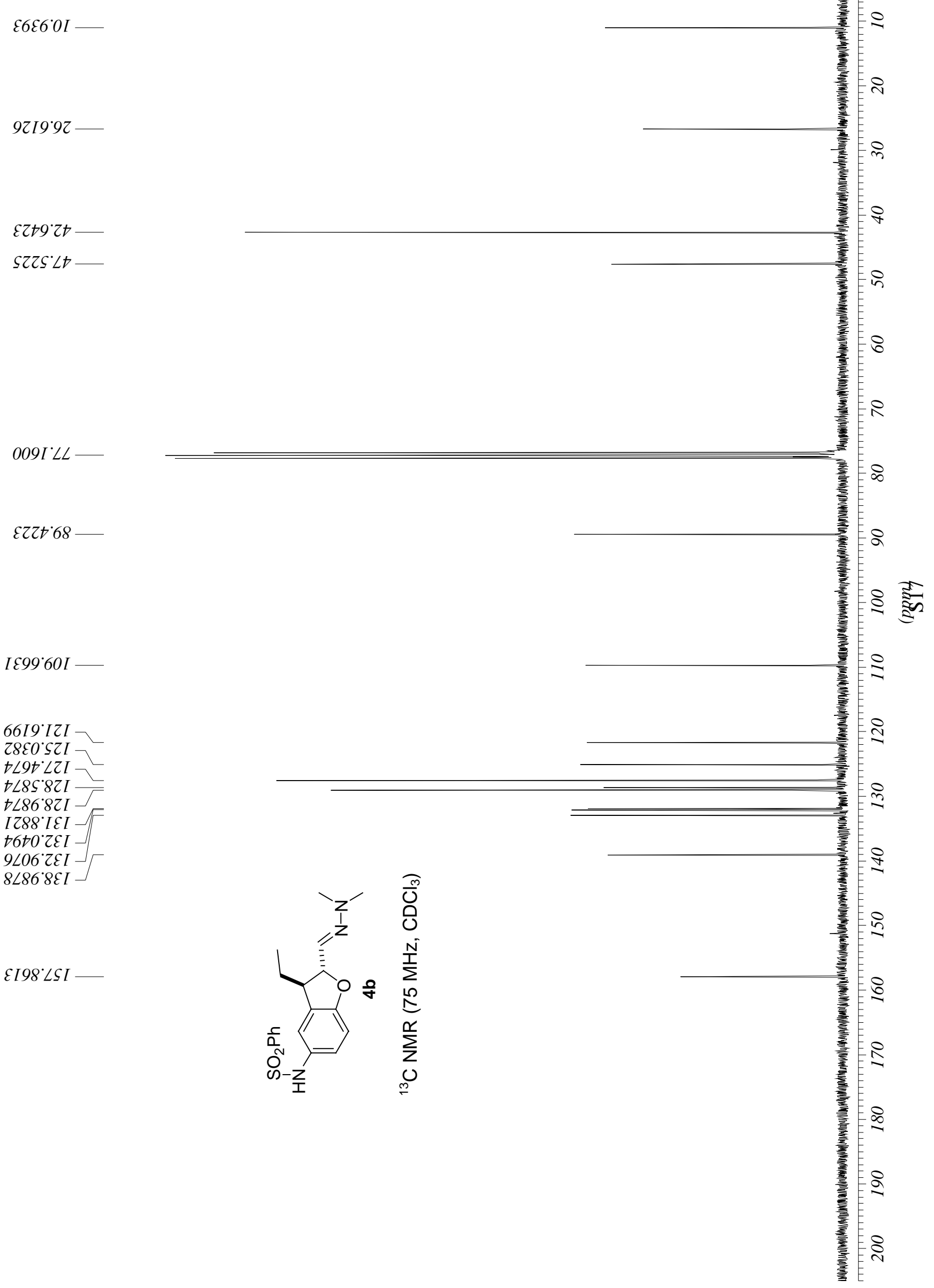


$26 \varepsilon 60^{\circ} 0 I$

9z19.92

Ezt9'zt

$t z z S^{\circ} \angle t$

zzz+56 -

OE99.60I -

86I9. IZI

I8EO $S Z I-$

$\varepsilon \angle 9 t^{\circ} \angle Z I$

t $\angle 866^{\circ} 8 Z I$

$\varepsilon 6+0 \cdot z \varepsilon I-F$

SLO6'ZEI -

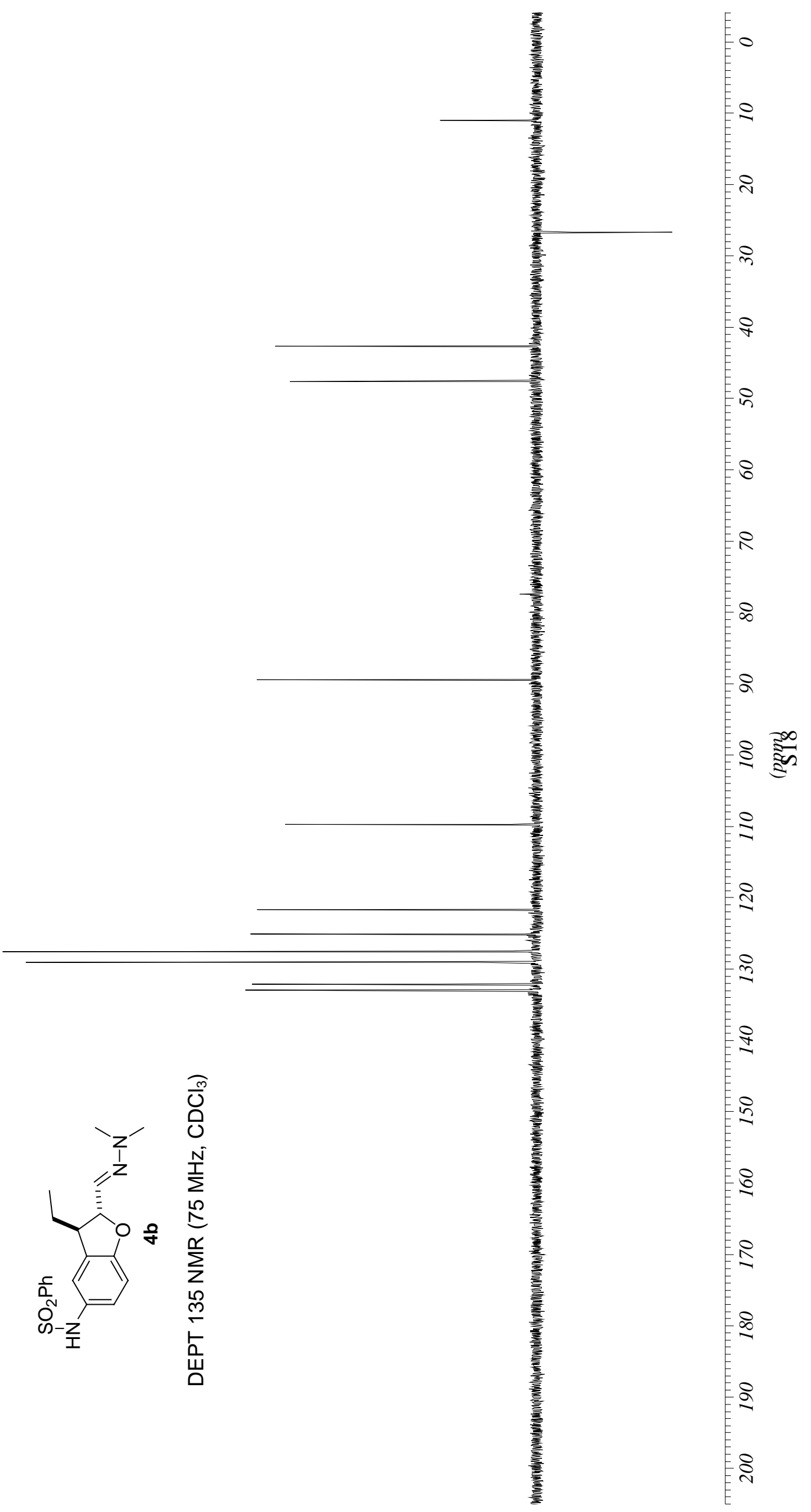




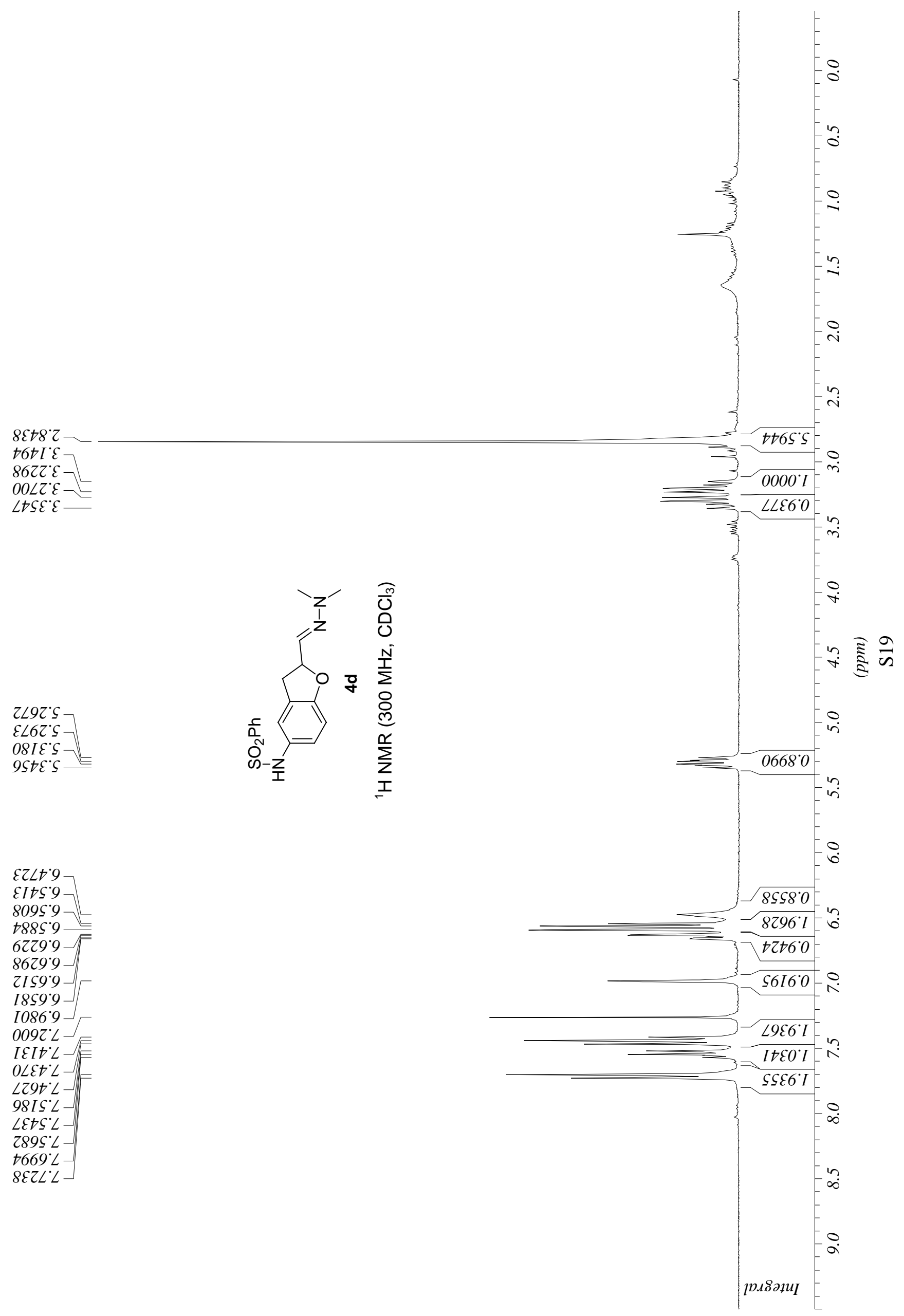




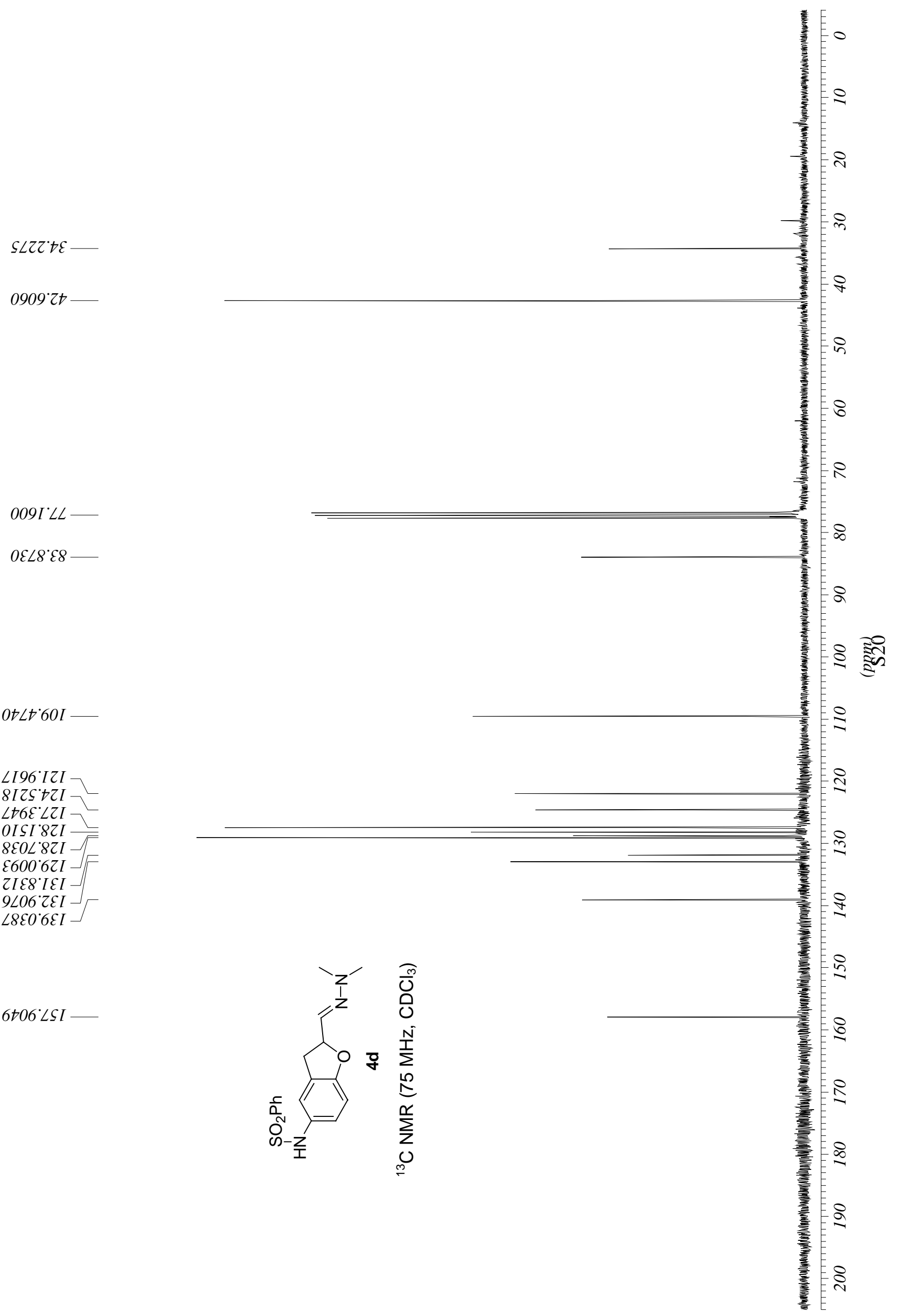




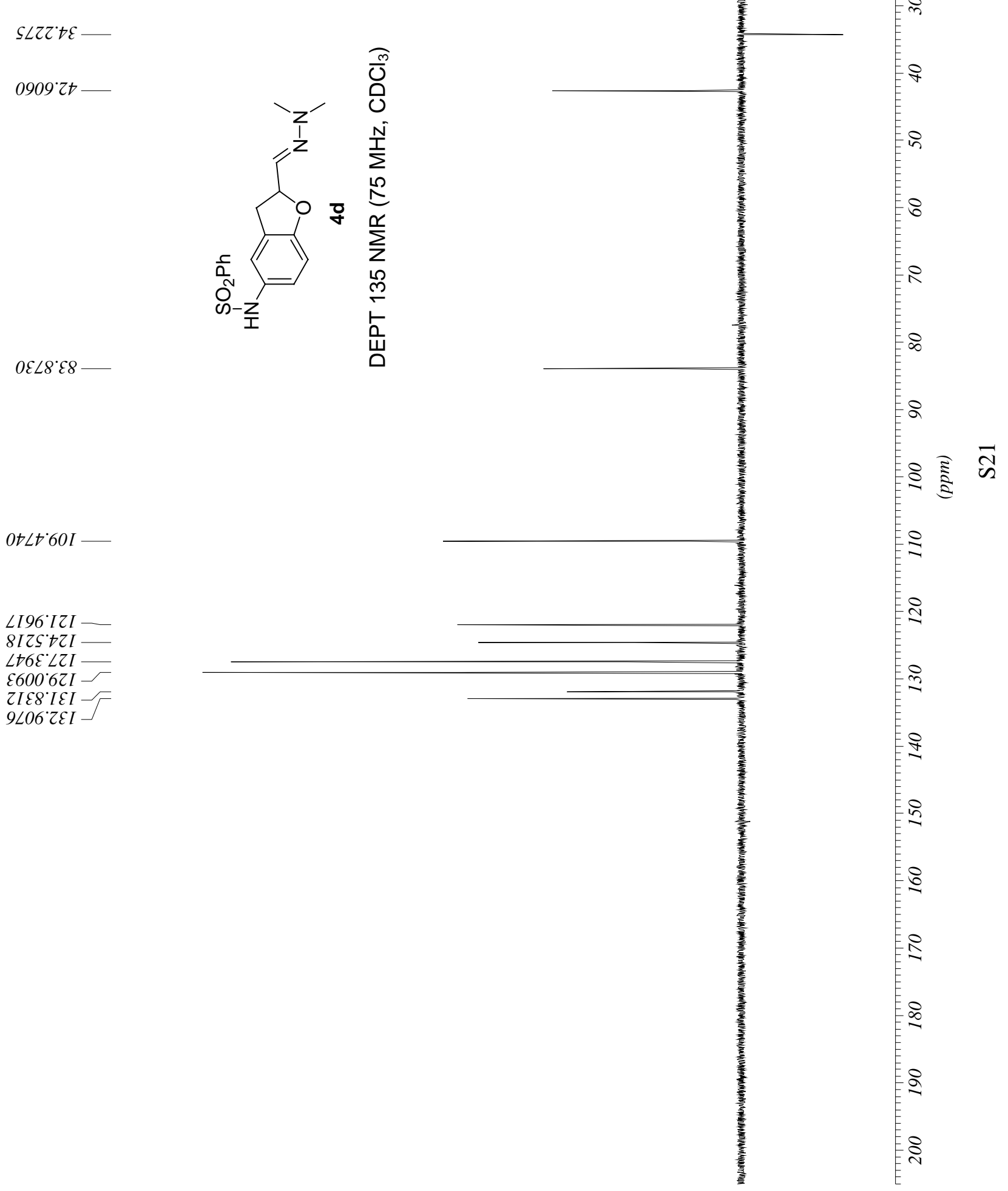




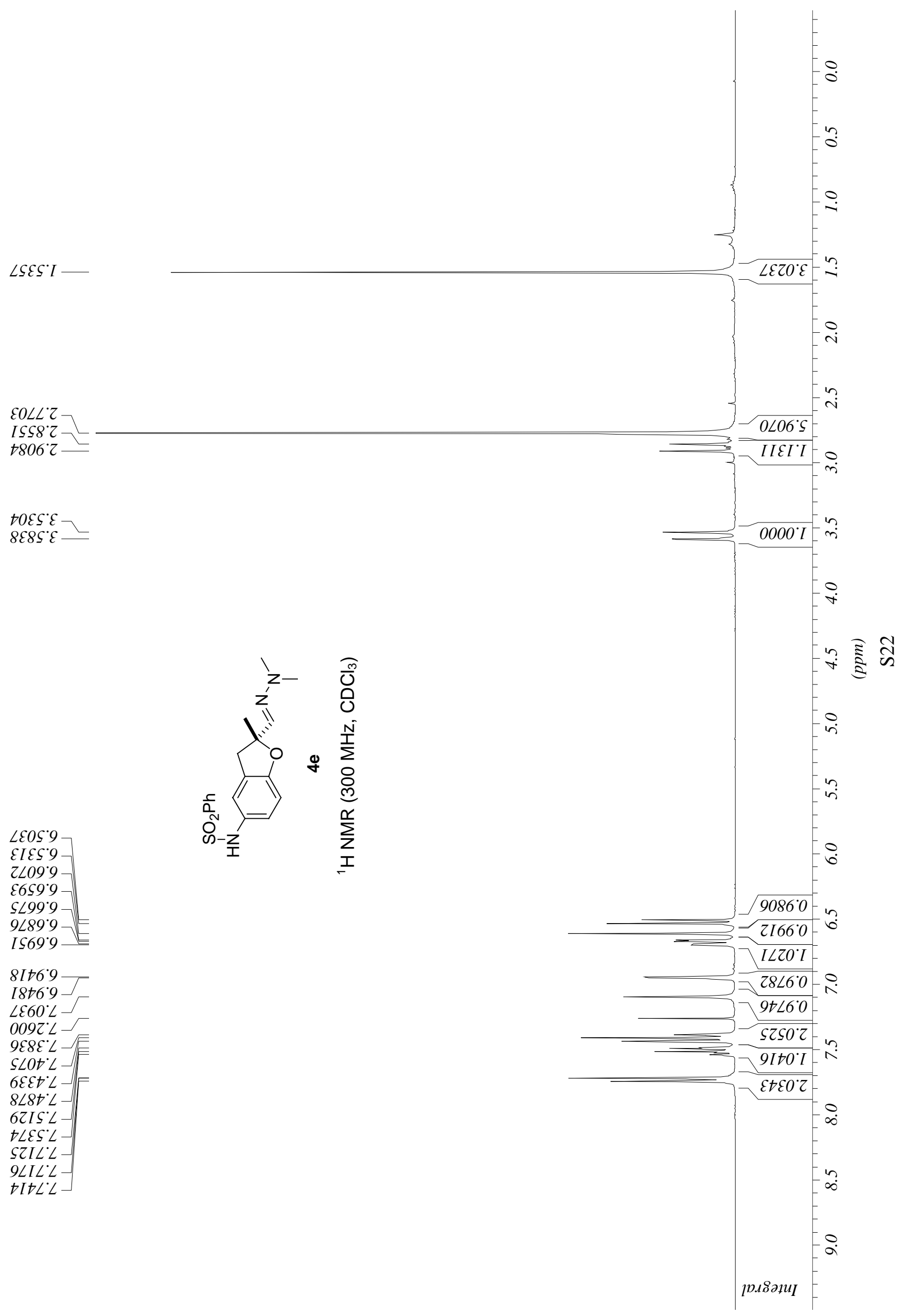


$97 \varsigma 9^{\circ} \varsigma 2$

$0+0 \angle \circ 6$

$\angle 8 S \angle \cdot Z$ -

$009 I^{\circ} L L$

$\varsigma 0+2 \cdot 68$

IZES $60 I$

$6660^{\circ} 22 I$

I69E'tZI

$\angle 8 \mathcal{E}^{\circ} \angle Z I-$

$\angle t \varepsilon t^{\circ} 8 z I-T$

$626 t^{\circ} 82 I$

I IS6 $82 I$

$6+\varepsilon 8^{\circ} \tau \varepsilon I$

$89 \mathcal{L} L \mathcal{S E I}-$

$\varepsilon \mathcal{E} 0^{\circ} 6 \mathcal{E} I$

$8566^{\circ} 9 S I$
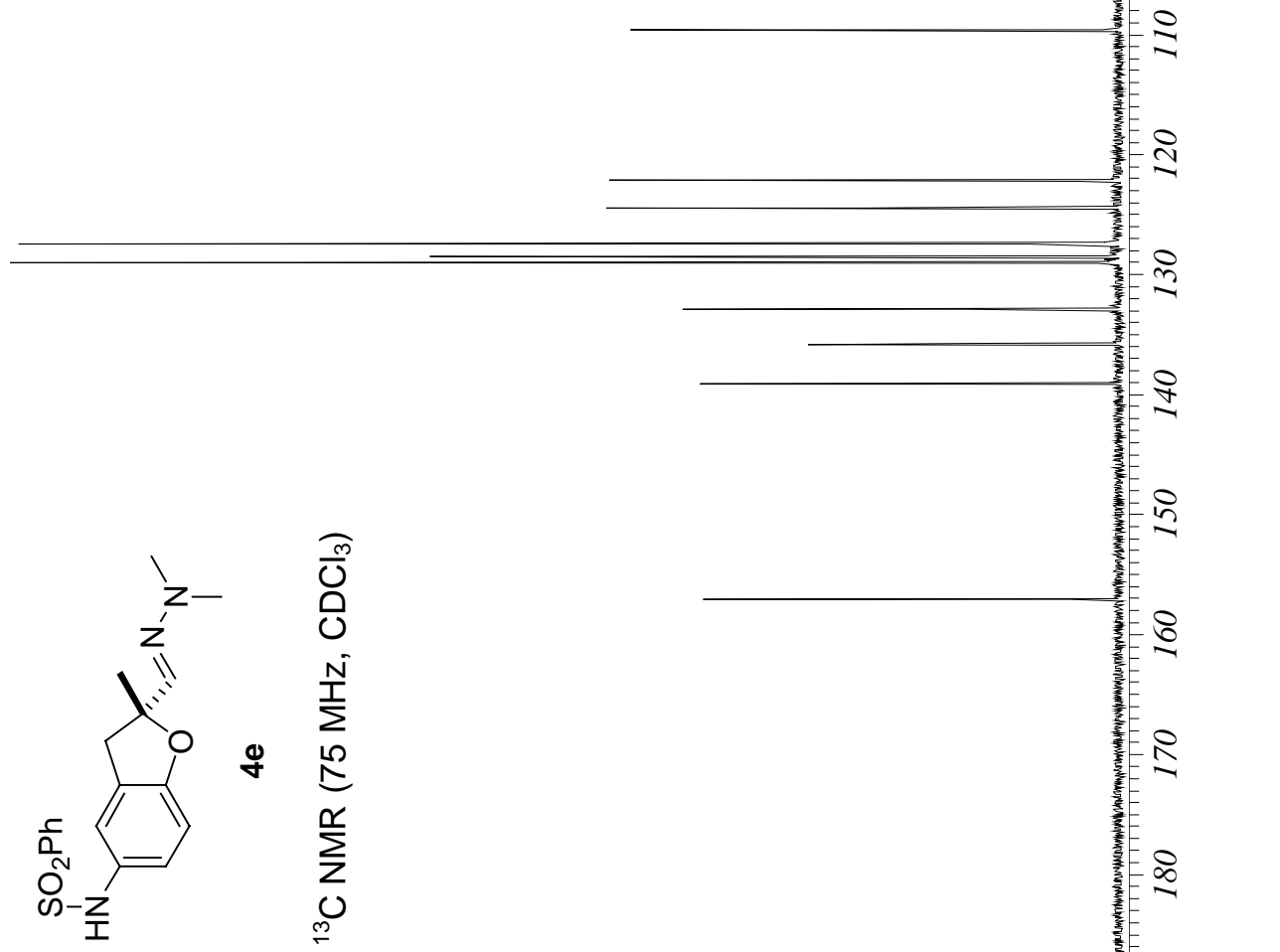


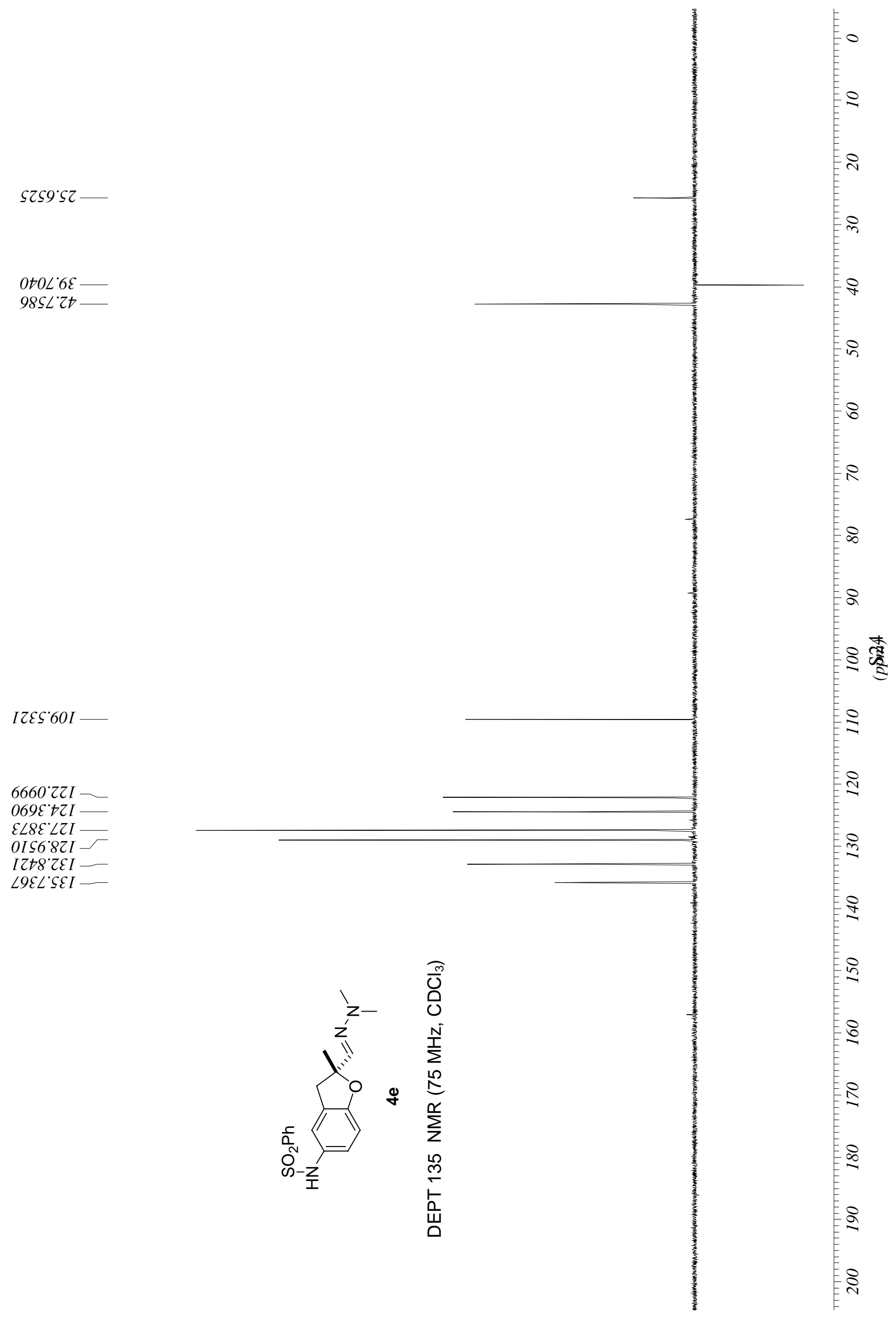


t $26 L^{\circ} I$

S888 $\mathrm{I} \longrightarrow$

IS86. I
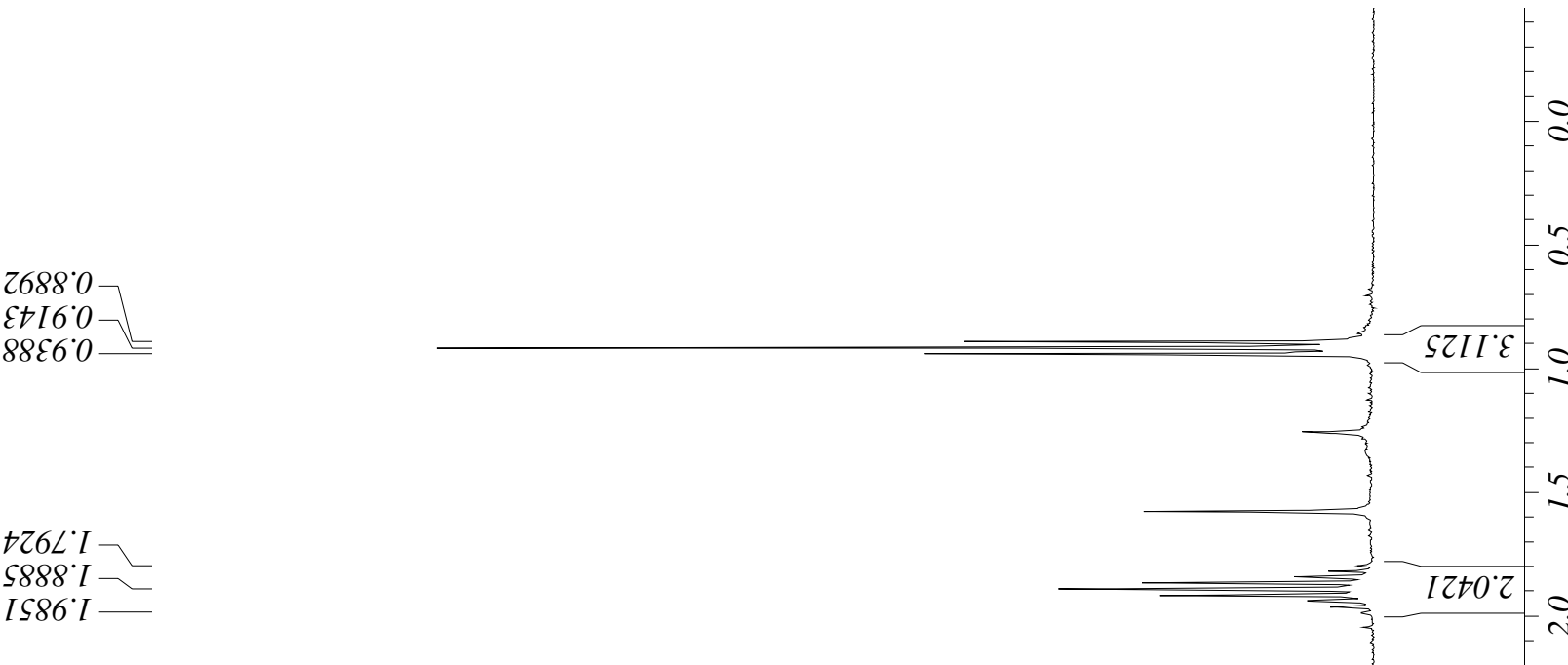

$\varepsilon Z 8 \angle{ }^{\circ} Z$

$8 \angle 68^{\circ} Z$

I IS6 2

$7919^{\circ} \mathcal{E}$

$8699^{\circ} \mathcal{E}$

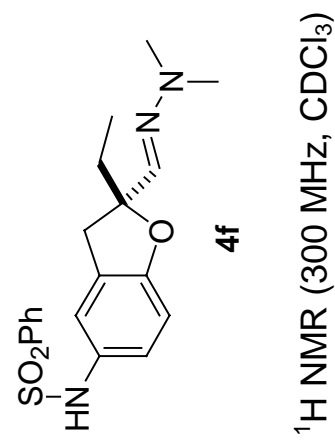

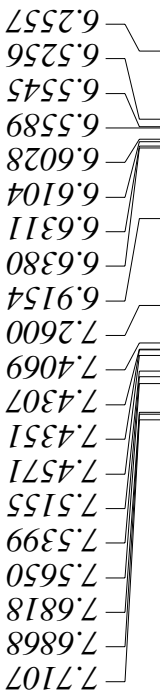

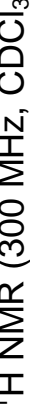

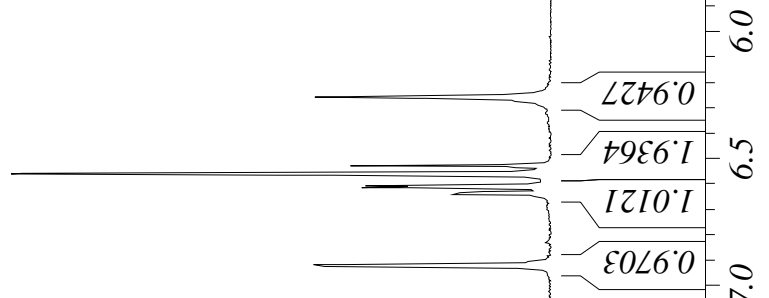

$\stackrel{\circ}{2}$

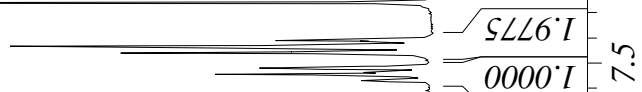

$+9 \varepsilon 6{ }^{\circ}$ 


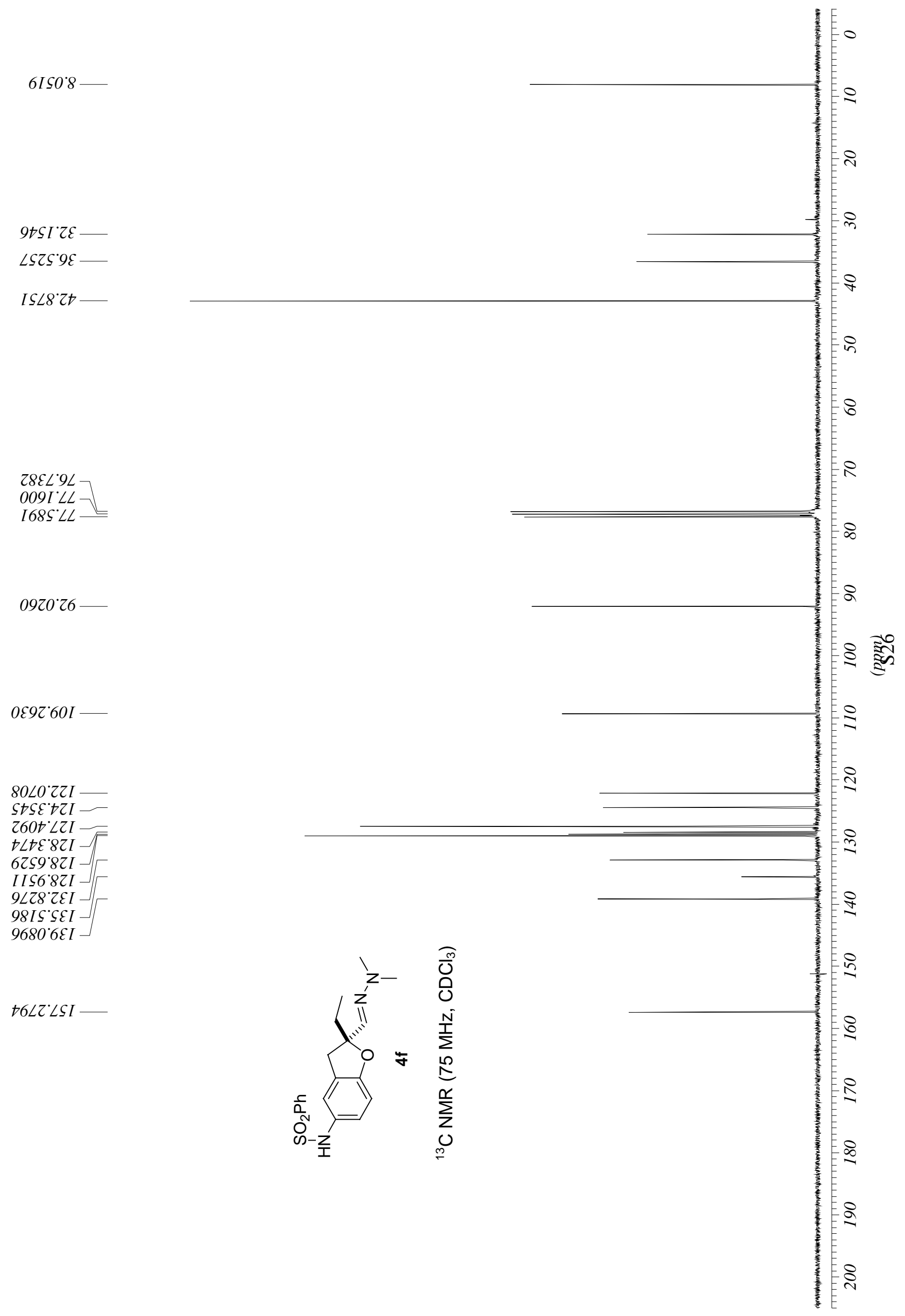




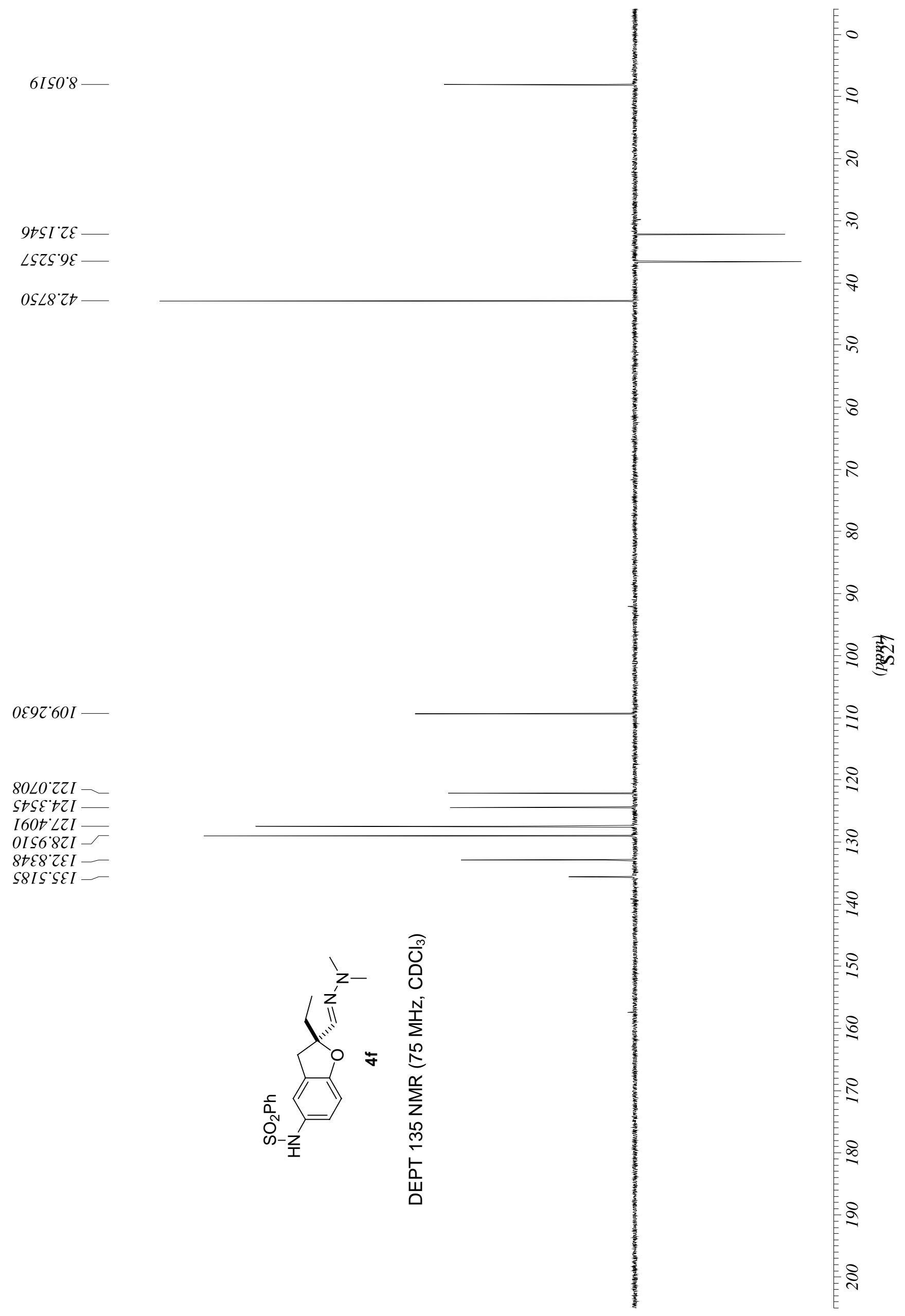


ZL9I' $\mathrm{Z}$ 9I8I' $\mathrm{Z}$ $6202 \cdot 2$ $+\angle I Z^{\circ} Z$ $\angle 9 S \mathcal{C}^{\circ} Z$

$t \hbar \neg t^{\circ} \varepsilon$ $8 \varepsilon t t^{\circ} \mathcal{E}$ $S 6 S t^{\circ} \mathcal{E}$ Z08t $\mathcal{}{ }^{\circ} \mathcal{E}$ $88 \angle S^{\circ} \mathcal{E}$ $0{ }^{\circ} 8 S^{\circ} \mathcal{E}$ $2 \varepsilon 6 S^{\circ} \mathcal{E}$ $\varsigma 66 \varsigma^{\circ} \mathcal{E}-$ $0 \mathcal{E} 89^{\circ} \mathcal{E}$

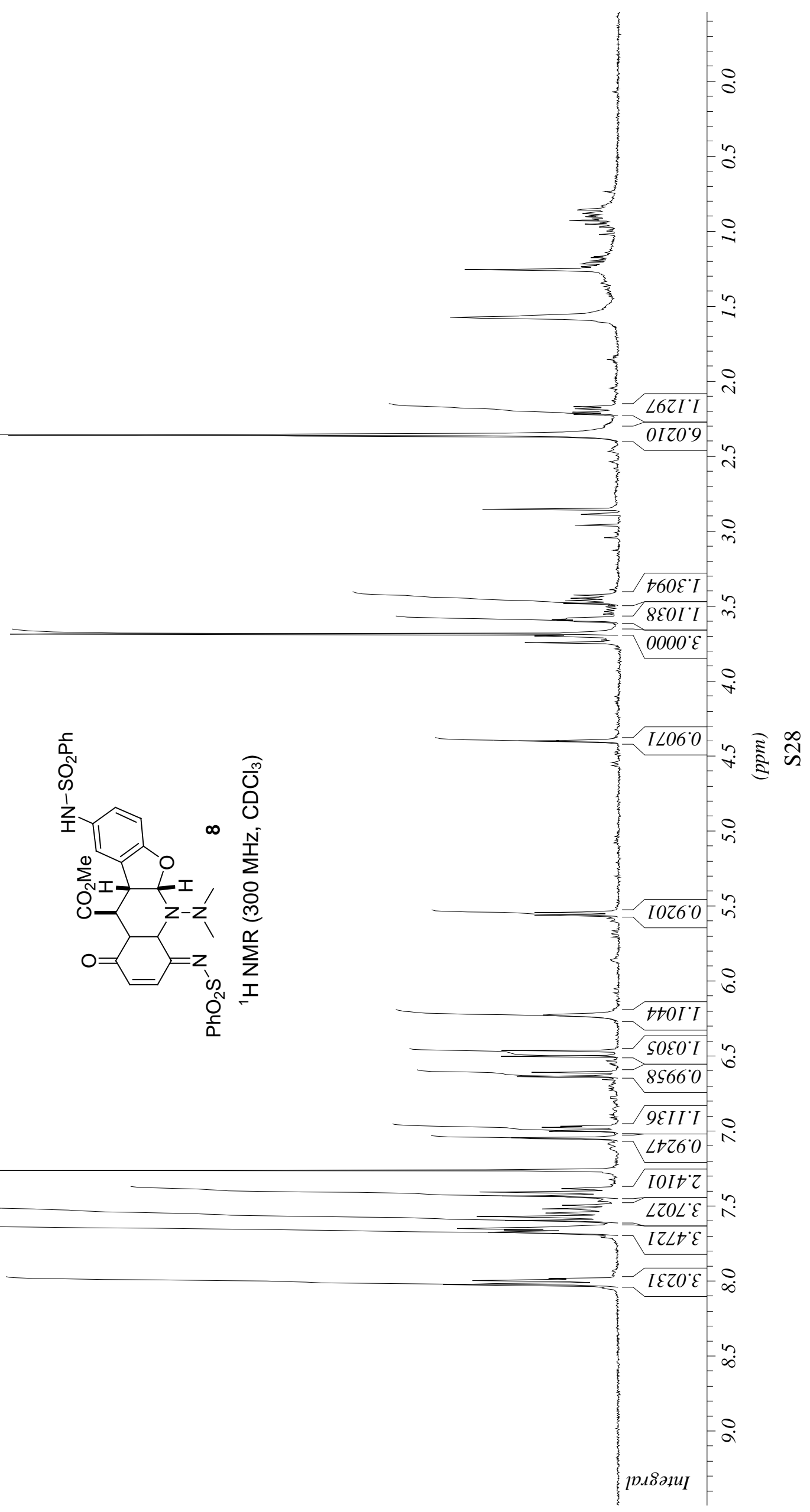

$\operatorname{EgES}^{\circ} \mathrm{S}$

$8 \angle S S^{\circ} \mathcal{S}$

$\tau \angle 6 \mathcal{E}^{\circ} \circ$

IEZ2 9

z29t' 9

$+\angle 60^{\circ} 9$

$\varepsilon S 09^{\circ} 9-$

OEE9 9

$\angle \varepsilon 96^{\circ} 9$

$6 I \angle 6^{\circ} 9$

$0266^{\circ} 9$

$1000^{\circ} \mathrm{L}$

$60+0 \% \mathrm{~L}$

I $6+00^{\circ} \mathrm{L}$

$0092^{\circ} \mathrm{L}$

SO8E $\mathrm{L}$

$t+0 t^{\circ} \mathrm{L}$

$\angle O E H^{\circ} \mathrm{L}$

$0 I 6 t^{\circ} \mathrm{L}$

$6 \varepsilon 6 S^{\circ} \mathrm{L}$

SEE9 $\mathrm{L}$

$8 I 89^{\circ} \mathrm{L}$

$2186^{\circ} \mathrm{L}$

$\varepsilon 686^{\circ} \mathrm{L}$

$9 S 66^{\circ} \mathrm{L}$

$02200^{\circ}$ 


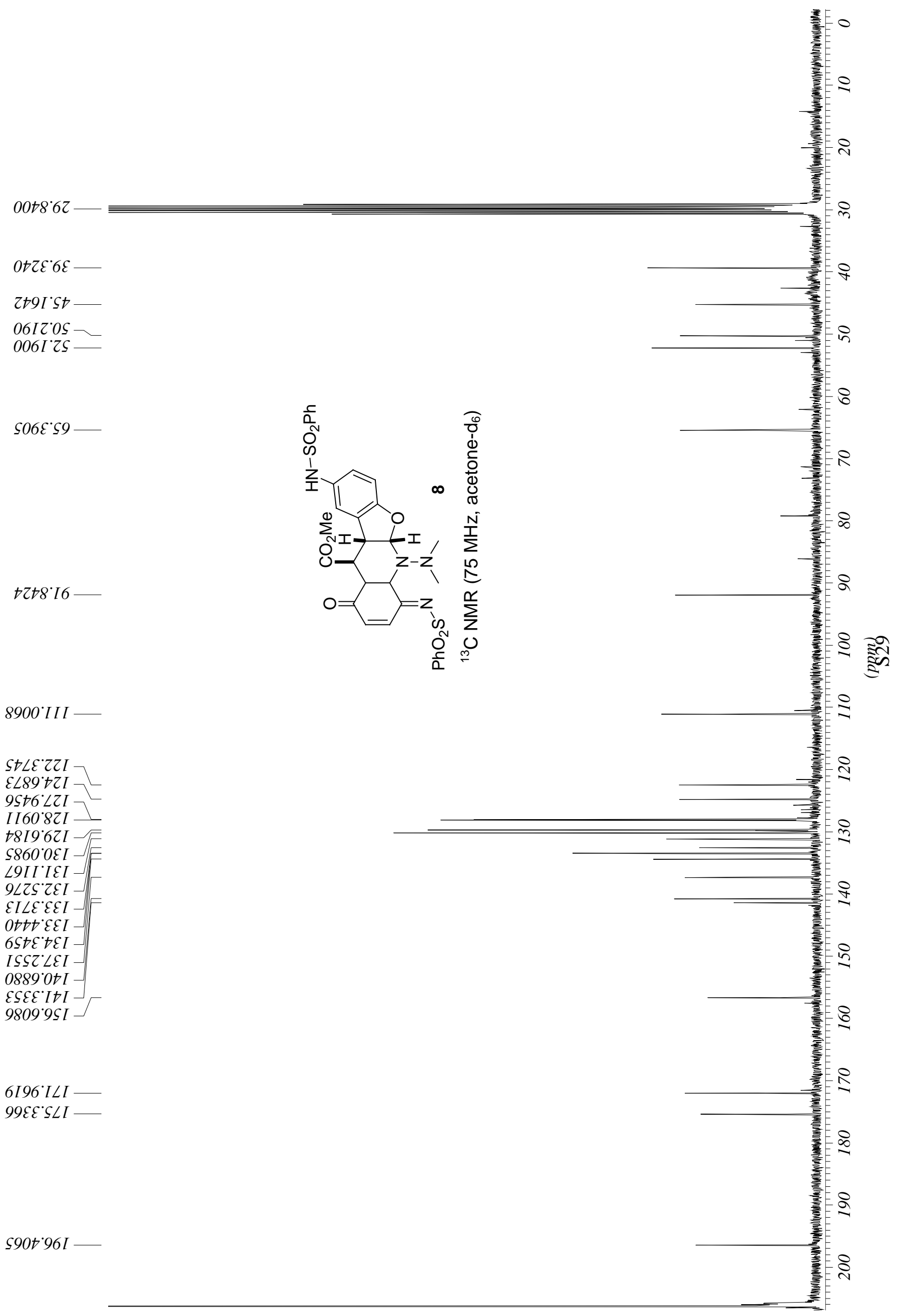




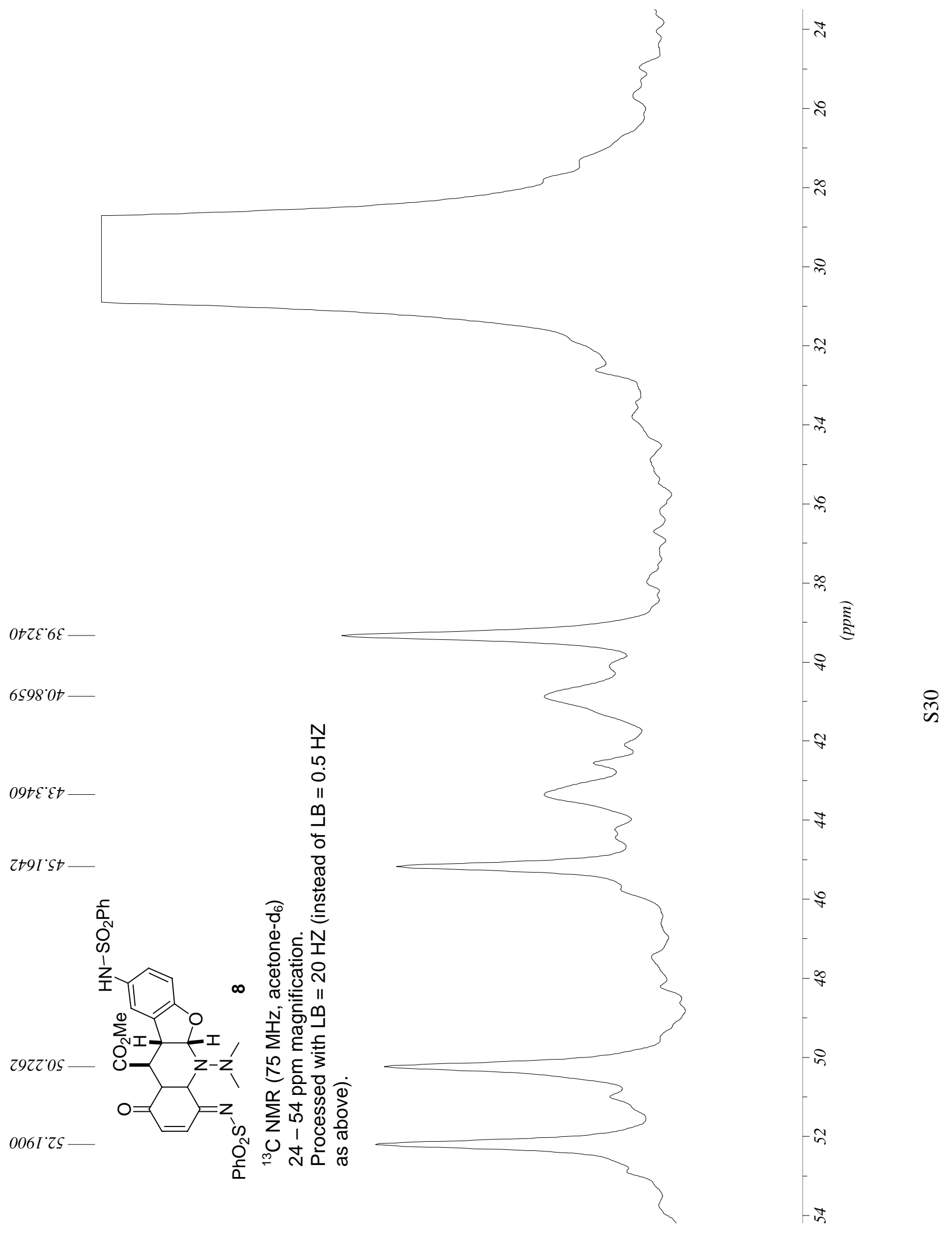



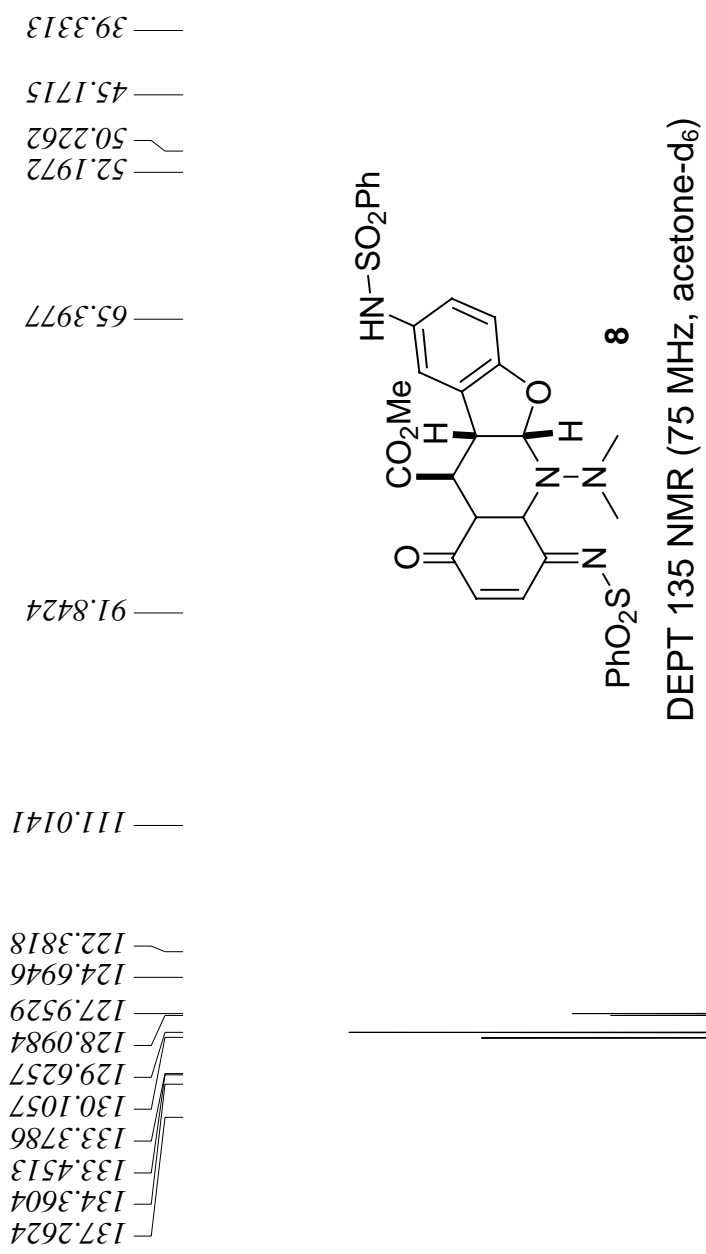

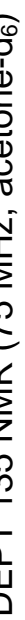

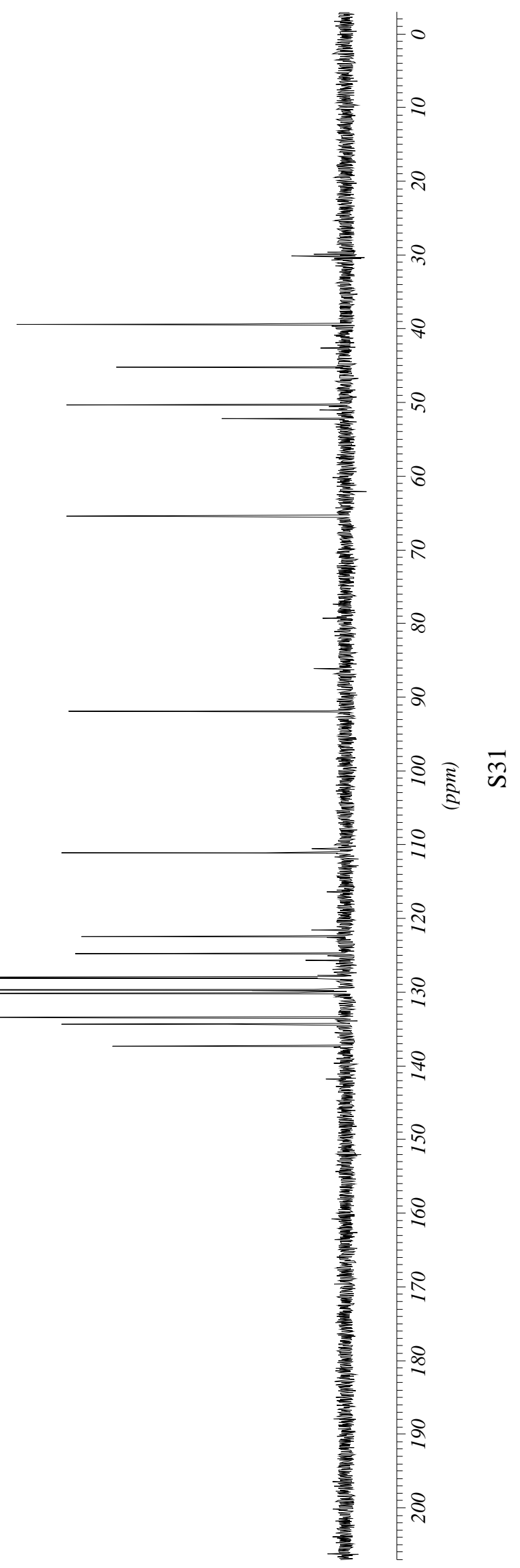

